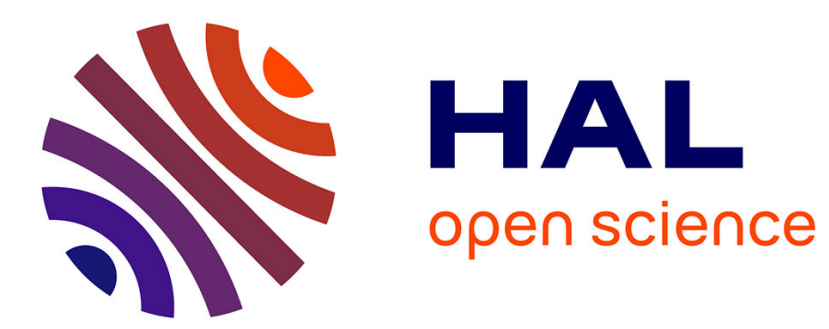

\title{
Geodesics in trees of hyperbolic and relatively hyperbolic spaces
}

François Gautero

\section{To cite this version:}

François Gautero. Geodesics in trees of hyperbolic and relatively hyperbolic spaces. 2011. hal00769023v1

\section{HAL Id: hal-00769023 \\ https://hal.science/hal-00769023v1}

Preprint submitted on 27 Dec 2012 (v1), last revised 20 Apr 2015 (v2)

HAL is a multi-disciplinary open access archive for the deposit and dissemination of scientific research documents, whether they are published or not. The documents may come from teaching and research institutions in France or abroad, or from public or private research centers.
L'archive ouverte pluridisciplinaire HAL, est destinée au dépôt et à la diffusion de documents scientifiques de niveau recherche, publiés ou non, émanant des établissements d'enseignement et de recherche français ou étrangers, des laboratoires publics ou privés. 


\title{
GEODESICS IN TREES OF HYPERBOLIC AND RELATIVELY HYPERBOLIC SPACES
}

\author{
FRANÇOIS GAUTERO
}

\begin{abstract}
We present a careful approximation of the quasi geodesics in trees of hyperbolic and relatively hyperbolic spaces. As an application we prove a dynamical and geometric combination theorem for trees of relatively hyperbolic spaces, with both Farb's and Gromov's definitions.
\end{abstract}

\section{INTRODUCTION}

The main part of this paper is devoted to giving a precise description of (quasi) geodesics in trees of hyperbolic and relatively hyperbolic spaces. As an application of this description we prove a combination theorem for such spaces. That is, a theorem giving a condition for a tree of relatively hyperbolic spaces being a relatively hyperbolic space. In [2], the authors introduce the notions of (finite) graphs of qi-embedded spaces. Assuming the Gromov hyperbolicity of the vertex spaces and the quasiconvexity of the edge spaces in the vertex spaces, they describe sufficient conditions for the universal covering of the given graph of qi-embedded spaces to be hyperbolic and then describe group-theoretic consequences. For related papers in a group-theoretic setting, see $[13,17,15,16]$. The paper [10] gives a new proof of [2] by an approach similar to the one presented here, in the case of mapping-tori of $\mathbb{R}$-trees (i.e. 0-hyperbolic spaces) whereas [12] treats the case of mapping-tori of surface homeomorphisms: this mapping-torus case is in some sense the prototype of the "non-acylindrical" case, which is actually the case where interesting phenomena appear.

Nowadays the attention has shifted from hyperbolic spaces to relatively hyperbolic spaces. A notion of relative hyperbolicity was already defined by Gromov in his seminal paper [14]. Since then it has been revisited and elaborated on in many papers. Two distinct definitions now coexist. In parallel to the Gromov relative hyperbolicity, sometimes called strong relative hyperbolicity, there is the notion of weak relative hyperbolicity introduced by Farb [9] (for alternative definitions in a group-theoretic setting see Bowditch [3] or Osin [20]). In fact, it has been proved [6, 20] (see also [3]) that Gromov's definition is equivalent to Farb's definition plus an additional property, due to Farb [9]. Relatively hyperbolic spaces in the strong (that is Gromov) sense form a class encompassing Gromov hyperbolic spaces, geometrically finite orbifolds with pinched negative curvature, CAT(0)-spaces with isolated flats among many others. First combination theorems, for group-theory inclined people, in some particular (essentially acylindrical) cases have been given in the setting of relative hyperbolicity: [1], [8] or [19, 21]. One more geometric result [12] treats a particular non-acylindrical case, namely the relative hyperbolicity of mapping-tori of surface homeomorphisms. In [18] the authors give a geometric combination theorem dealing with trees of relatively hyperbolic spaces. It heavily relies

Date: December 7, 2012.

2000 Mathematics Subject Classification. 20F65, 57M50.

Key words and phrases. Gromov-hyperbolicity, Farb and Gromov relative hyperbolicity, trees of spaces, mapping-tori, combination theorem. 
upon [2], which is used as a "black-box". In the current paper, as an application of our work on geodesics in trees of spaces we offer a quite general combination theorem for relatively hyperbolic spaces. We emphasize at once that we do not appeal to [2], but instead give a new proof of it as a particular case. Where the authors of [2] use "second-order" geometric characterization of hyperbolicity via isoperimetric inequalities, we use "first-order" geometric characterization, via approximations of geodesics and the thin triangle property. At the expense of heavier and sometimes tedious computations, this naive approach allows us to simultaneously deal with both absolute and relative hyperbolicity.

The group-theoretic consequences of the geometric combination theorem we prove here have been postponed to another paper. The first versions of this work, which go back to 2005 (and were presented in 2006 for the defense of the habilitation thesis of the author [11]), included them: geometry and group-theory were intimately linked, which at some points caused some unnecessary complications and vague formulations. R. Weidmann pointed out the needed clarifications, which lead on the one hand to a clear statement of a geometric and dynamical combination theorem (the result of the current paper), and on the other hand to a much more general group-theoretic result. This is why we chose to separate the two points of view.

Acknowledgements. Warm thanks are due to I. Kapovich (Urbana-Champaign) for his numerous explanations about the combination theorem. At that time, the author was Assistant at the University of Geneva, whereas I. Kapovich visited this university thanks to a funding of the Swiss National Science Foundation. The author is glad to acknowledge support of these institutions, as well as the support of the University Blaise Pascal (Clermont-Ferrand) where this work was finalized. Professor P. de la Harpe (Geneva) also deserves a great share of these acknowledgements for his help. Last but not least $\mathrm{R}$. Weidmann (Kiel) and the referee provided an invaluable help to correct some mistakes and get a better presentation.

\section{Statements of Results}

We begin with recalling basic definitions about coarse geometry, and in particular Gromov hyperbolicity.

A $(\lambda, \mu)$-quasi isometric embedding from a metric space $\left(X_{1}, d_{1}\right)$ to a metric space $\left(X_{2}, d_{2}\right)$ is a map $f: X_{1} \rightarrow X_{2}$ such that, for any $x, y$ in $X_{1}$ :

$$
\frac{1}{\lambda} d_{1}(x, y)-\mu \leq d_{2}(f(x), f(y)) \leq \lambda d_{1}(x, y)+\mu
$$

A $(\lambda, \mu)$-quasi isometry $f:\left(X_{1}, d_{1}\right) \rightarrow\left(X_{2}, d_{2}\right)$ is a $(\lambda, \mu)$-quasi isometric embedding such that for any $y \in X_{2}$ there exists $x \in X_{1}$ with $d_{2}(f(x), y) \leq \mu$.

A $(\lambda, \mu)$-quasi geodesic in a metric space $(X, d)$ is the image of an interval of the real line under a $(\lambda, \mu)$-quasi isometric embedding.

Since quasi isometric embeddings are not necessarily continuous, a quasi geodesic as defined above is not a path in the usual sense. A geodesic is a $(1,0)$-quasi geodesic. We denote by $[x, y]$ any geodesic between two points $x$ and $y$ in a metric space $(X, d)$. A geodesic space is a metric space in which there exists (at least) one geodesic between any two points. We will need the slightly more general notion of quasi geodesic space: a $(r, s)$-quasi geodesic space is a metric space $(X, d)$ in which there exists (at least) one $(r, s)$-quasi geodesic between any points; a quasi geodesic space is a metric space which is a $(r, s)$-quasi geodesic space for some constants $r \geq 1$ and $s \geq 0$. 
We work with a version of Gromov hyperbolic spaces which is slightly extended with respect to the one most commonly used by not requiring properness, that is closed balls are not necessarily compact. Not requiring our spaces to be proper is important in order to deal with relatively hyperbolic spaces, the definitions of which involve non-proper metric spaces. A geodesic triangle in a metric space $(X, d)$ is $\delta$-thin if and only if any side is contained in the $\delta$-neighborhood of the union of the two other sides. (Quasi) geodesic triangles in a (quasi) geodesic metric space $(X, d)$ are thin if there exists $\delta \geq 0$ such that all (quasi) geodesic triangles are $\delta$-thin (of course in the case of a quasi geodesic space the constant $\delta$ depends on the constants of quasi geodesicity - denoted $r, s$ above). In this case, $X$ is a $\delta$-hyperbolic space. A metric space $(X, d)$ is a Gromov hyperbolic space if and only if there exists $\delta \geq 0$ such that $(X, d)$ is a $\delta$-hyperbolic space.

We now recall the definitions of weak and strong relative hyperbolicity. Both notions were defined in [9]. If $S$ is a set, the cone with base $S$ is the space $S \times\left[0, \frac{1}{2}\right]$ with $S \times\{0\}$ collapsed to a point, termed the vertex of the cone or cone-vertex. This cone is considered as a metric space, with distance function $d_{S}\left((x, t),\left(y, t^{\prime}\right)\right)=t+t^{\prime}$ if $x \neq y$ and $d_{S}\left((x, t),\left(x, t^{\prime}\right)\right)=\left|t-t^{\prime}\right|$. Let $(X, d)$ be a geodesic space. Putting a cone over a closed subset $S$ of $X$ consists of pasting to $X$ a cone with base $S$ by identifying $S \times\{1 / 2\}$ with $S \subset X$. The resulting metric space (i.e. the metric is the quotient metric - see [5][§5.18]) is denoted by $\widehat{X}$ and its subspace consisting of the cone over $S$ by $\widehat{S}$. The space $\widehat{X}$ is such that all the points in $S$ are now at distance $\frac{1}{2}$ from the cone-vertex and so at distance at most 1 one from each other.

\section{Definition 2.1.}

A geodesic pair $(X, \mathcal{P})$ is a geodesic space $X$ equipped with a family of disjoint closed subspaces $\mathcal{P}=\left\{P_{i}\right\}_{i \in \Lambda}$, termed parabolic subspaces, which are geodesic subspaces with respect to the induced path-metric.

The induced path-metric on $P_{i}$ is the path-metric obtained by defining the distance between two points in $P_{i}$ as the infimum of the lengths of the paths in $P_{i}$ between these two points, the length being measured with respect to the metric of $X$. We could only require the parabolic subspaces to be quasi geodesic subspaces, the adaptations thereafter are straightforward.

\section{Definition 2.2. [9]}

Let $(X, \mathcal{P})$ be a geodesic pair.

(a) The coned-space $\left(\widehat{X}_{\mathcal{P}}, d_{\mathcal{P}}\right)$ is the metric space obtained from $(X, \mathcal{P})$ by putting a cone over each parabolic subspace in $\mathcal{P}$ and $d_{\mathcal{P}}$ is the coned, or relative distance.

(b) The space $X$ is weakly hyperbolic relative to $\mathcal{P}$ if and only if the coned-space $\left(\widehat{X}, d_{\mathcal{P}}\right)$ is Gromov hyperbolic.

Let $\left(\widehat{X}_{\mathcal{P}}, d_{\mathcal{P}}\right)$ be a coned-space. We say that a path $\widehat{g}$ in $\widehat{X}$ backtracks if for the arclength parametrization of $g:[0, l] \rightarrow \widehat{X}$ there exists a parabolic subspace $P_{i}$ and times $0 \leq t_{0}<t_{1}<t_{2} \leq l$ such that $g\left(t_{0}\right) \in P_{i}, g\left(t_{2}\right) \in P_{i}$ and $g\left(t_{1}\right) \notin P_{i}$. In other words a path backtracks if and only if it reenters a parabolic subspace that it left before. Let $\widehat{g}$ be a $(u, v)$-quasi geodesic path in $\left(\widehat{X}_{\mathcal{P}}, d_{\mathcal{P}}\right)$ which does not backtrack. A trace $g$ of $\widehat{g}$ is a subpath of $X$ obtained by substituting each subpath of $\widehat{g}$ in the complement of $X$ by a subpath in some parabolic subspace $P_{i}$, which is a geodesic for the path-metric induced by $X$ on $P_{i}$.

Definition 2.3. [9] Let $(X, \mathcal{P})$ be a geodesic pair. 
The coned-space $\left(\widehat{X}_{\mathcal{P}}, d_{\mathcal{P}}\right)$ satisfies the Bounded-Parabolic Penetration property (BPP) if and only if there exists $C(u, v) \geq 0$ such that, for any two $(u, v)$-quasi geodesics $\widehat{g}_{0}, \widehat{g}_{1}$ of $\left(\widehat{X}_{\mathcal{P}}, d_{\mathcal{P}}\right)$ with traces $g_{0}, g_{1}$ in $(X, d)$, which have the same initial point, which have terminal points at most one apart and which do not backtrack, the following two properties are satisfied:

(a) if both $g_{0}$ and $g_{1}$ intersects a parabolic subspace $P_{i}$ then their first intersection points with $P_{i}$ are $C(u, v)$-close in $(X, d)$,

(b) if $g_{0}$ intersects a parabolic subspace $P_{i}$ and $g_{1}$ does not intersect $P_{i}$, then the diameter in $(X, d)$ of $g_{0} \cap P_{i}$ is bounded above by $C(u, v)$.

Definition 2.4. [9] Let $(X, \mathcal{P})$ be a geodesic pair.

The space $X$ is strongly hyperbolic relative to $\mathcal{P}$ if and only if the coned-space $\left(\widehat{X}_{\mathcal{P}}, d_{\mathcal{P}}\right)$ is Gromov hyperbolic and satisfies the BPP.

Since the ultimate goal is a theorem about trees of relatively hyperbolic spaces, we introduce some notations for graphs and graphs of spaces. If $\Gamma$ is a graph, $V(\Gamma)$ (resp. $E(\Gamma)$ ) denotes its set of vertices (resp. of oriented edges). For $e \in E(\Gamma)$ we denote by $e^{-1}$ the same edge with opposite orientation. The map $e \mapsto e^{-1}$ is a fixed-point free involution of $E(\Gamma)$. If $p$ is an edge-path in $\Gamma$, in particular if $p$ is an edge, $i(p)$ (resp. $t(p)$ ) denotes the initial (resp. terminal) vertex of $p$. An edge-path $p$ is reduced if no edge $e$ in $p$ is followed by its opposite $e^{-1}$. In a tree, given any two vertices $x, y$, we denote by $[x, y]$ the unique reduced edge-path from $x$ to $y$. A metric tree $T$ is a tree equipped with a length one on each edge $e$ and an isometry from $e$ to the real interval $(0,1)$. If $p$ is a path in a metric tree $T$ then $|p|_{T}$ denotes the length of $p$ in $T$, whereas $d_{T}(x, y) \equiv|[x, y]|_{T}$ denotes the geodesic distance between any two points $x, y$ in $T$.

Definition 2.5. (compare [2])

(a) A tree of geodesic spaces $\mathfrak{T}=\left(\mathcal{T},\left\{X_{e}\right\},\left\{X_{v}\right\},\left\{\jmath_{e}\right\}\right)$ is a metric tree $\mathcal{T}$ with length 1 edges, together with two collections of geodesic spaces, the collection of edgespaces $\left\{X_{e}\right\}_{e \in E(\Gamma)}$ indexed over the oriented edges $e$ of $\mathcal{T}$ which satisfy $X_{e}=X_{e^{-1}}$ and the collection of vertex-spaces $\left\{X_{v}\right\}_{v \in V(\Gamma)}$ indexed over the vertices $v$ of $\mathcal{T}$, and a collection of maps $\jmath_{e}: X_{e} \rightarrow X_{t(e)}$ from the edge-spaces to the vertex-spaces.

(b) A tree of qi-embedded geodesic spaces is a tree of geodesic spaces $\left(\mathcal{T},\left\{X_{e}\right\},\left\{X_{v}\right\},\left\{\jmath_{e}\right\}\right)$ such that there exist two fixed real constants $\mathfrak{a} \geq 1$ and $\mathfrak{b} \geq 0$ such that the maps $\jmath_{e}: X_{e} \rightarrow X_{t(e)}$ from the edge-spaces $X_{e}$ to the vertex-spaces $X_{v}$ are $(\mathfrak{a}, \mathfrak{b})$-quasi isometric embeddings.

(c) A tree of hyperbolic spaces is a tree of qi-embedded geodesic spaces such that there is $\delta \geq 0$ for which each edge- and vertex-space is a $\delta$-hyperbolic space.

Of course, we could only require that the edge- and vertex-spaces be quasi geodesic spaces instead of geodesic ones, the adaptations are once again straightforward. Before defining trees of relatively hyperbolic spaces we need to introduce the notion of the conedextension of a map between geodesic pairs.

Definition 2.6. Let $(X, \mathcal{P})$ and $(Y, \mathcal{Q})$ be two geodesic pairs.

(a) A map $f: X \rightarrow Y$ is a pair-map from $(X, \mathcal{P})$ to $(Y, \mathcal{Q})$ if and only if for every parabolic subspace $P \in \mathcal{P}$ there is a unique parabolic subspace $Q \in \mathcal{Q}$ such that $f(P) \subset Q$.

(b) Let $f:(X, \mathcal{P}) \rightarrow(Y, \mathcal{Q})$ be a pair-map and let $\widehat{X}, \widehat{Y}$ be the coned-spaces associated to $(X, \mathcal{P})$ and $(Y, \mathcal{Q})$ respectively. A map $\widehat{f}: \widehat{X} \rightarrow \widehat{Y}$ is a coned-extension of $f$ if and only if it satisfies the following properties: 
- Its restriction to $X$ is equal to $f$.

- For any parabolic subspace $P \in \mathcal{P}$ with $f(P) \subset Q \subset \widehat{Q}, \widehat{f}$ is a pair-map from $(\widehat{X}, \widehat{P} \backslash P)$ to $(\widehat{Y}, \widehat{Q} \backslash Q)$ which sends the cone-vertex of $\widehat{P}$ to the cone-vertex of $\widehat{Q}$.

\section{Definition 2.7.}

(a) A tree of geodesic pairs $\left(\mathcal{T},\left\{\left(X_{e}, \mathcal{P}_{e}\right)\right\},\left\{\left(X_{v}, \mathcal{P}_{v}\right)\right\},\left\{\jmath_{e}\right\}\right)$ is a tree of geodesic spaces $\left(\mathcal{T},\left\{X_{e}\right\},\left\{X_{v}\right\},\left\{\jmath_{e}\right\}\right)$ such that for each edge $e$ and each vertex $v,\left(X_{e}, \mathcal{P}_{e}\right)$ and $\left(X_{v}, \mathcal{P}_{v}\right)$ are geodesic pairs, for each edge $e, \mathcal{P}_{e}=\mathcal{P}_{e^{-1}}$ and $\jmath_{e}:\left(X_{e}, \mathcal{P}_{e}\right) \rightarrow$ $\left(X_{t(e)}, \mathcal{P}_{t(e)}\right)$ is a pair-map.

(b) A tree of weakly (resp. strongly) relatively hyperbolic spaces is a tree of geodesic pairs $\mathfrak{T}=\left(\mathcal{T},\left\{\left(X_{e}, \mathcal{P}_{e}\right)\right\},\left\{\left(X_{v}, \mathcal{P}_{v}\right)\right\},\left\{\jmath_{e}\right\}\right)$ such that:

- For each edge $e$, the edge-space $X_{e}$ is weakly (resp. strongly) hyperbolic relatively to the family of parabolic subspaces $\mathcal{P}_{e}$. For each vertex $v$ the vertex-space $X_{v}$ is weakly (resp. strongly) hyperbolic relative to the family of parabolic subspaces $\mathcal{P}_{v}$.

- If $\widehat{X}_{e}$ and $\widehat{X}_{v}$ denote the coned-spaces equipped with the relative metrics associated to the geodesic pairs $\left(X_{e}, \mathcal{P}_{e}\right)$ and $\left(X_{v}, \mathcal{P}_{v}\right)$ and $\widehat{\jmath}_{e}$ is a coned-extension of $\jmath_{e}$ then $\widehat{\mathfrak{T}}=\left(\mathcal{T},\left\{\widehat{X}_{e}\right\},\left\{\widehat{X}_{v}\right\},\left\{\widehat{\jmath}_{e}\right\}\right)$ is a tree of qi-embedded geodesic spaces.

Remark 2.8. Our definition is more general than the corresponding definition in [18] because we do not require that the attaching-maps of the edge-spaces to the vertex-spaces be quasi isometric embeddings for the absolute metrics but only for the relative metrics.

Definition 2.9. Let $\mathfrak{T}=\left(\mathcal{T},\left\{X_{e}\right\},\left\{X_{v}\right\},\left\{\jmath_{e}\right\}\right)$ be a tree of geodesic spaces.

If $E^{+}(\mathcal{T})$ denotes the subset of $E(\mathcal{T})$ composed of exactly one representative in each pair $\left(e, e^{-1}\right)$ then the space $\widetilde{X}$ obtained from

$$
\bigsqcup_{e \in E^{+}(\mathcal{T})}\left(X_{e} \times[0,1]\right) \sqcup \bigsqcup_{v \in V(\mathcal{T})} X_{v}
$$

by identifying $(x, 1) \in X_{e} \times[0,1]$ with $\jmath_{e}(x) \in X_{t(e)}$ and $(x, 0) \in X_{e} \times[0,1]$ with $\jmath_{e^{-1}}(x) \in$ $X_{i(e)}$ for each $e \in E^{+}(\mathcal{T})$ is called the geometric realization of $\mathfrak{T}$.

We denote by $\pi: \tilde{X} \rightarrow \mathcal{T}$ the map which identifies each subset $X_{e} \times\{t\} \subset \widetilde{X}$ with the point in $e \in E(\mathcal{T})$ with coordinate $t \in[0,1]$ (recall that each edge $e$ comes with an isometry with $[0,1])$ and each subset $X_{v} \subset \widetilde{X}$ with the vertex $v$ of $\mathcal{T}$. The sets $X_{e} \times\{t\}$ with $t \in(0,1)$ and $X_{v}$ are the strata of $\widetilde{X}$. A path contained in a stratum is a horizontal path. By definition, each stratum in a tree of qi-embedded geodesic spaces comes with a metric. The associated length function defined on horizontal paths is termed horizontal length and the horizontal length of a horizontal path is denoted by $|p|_{\text {hor }}$. Similarly, the distance function associated to the metric of a stratum, which is defined for any two points in this stratum, is termed horizontal distance and the horizontal distance between any two points $x, y$ in a same stratum is denoted by $d_{\text {hor }}(x, y)$. Each subset $\{x\} \times[0,1]$, $x \in X_{e}$ for some edge $e$, also has its natural metric, the usual metric on $[0,1]$, which gives the notion of interval-length for subpaths contained in such subsets.

Definition 2.10. Let $(\widetilde{X}, \pi, \mathcal{T})$ be the geometric realization of a tree of qi-embedded geodesic spaces. For any two points $x, y$ in $\widetilde{X}$, let $\mathcal{P}(x, y)$ be the set of all the continuous paths from $x$ to $y$ which are the concatenation of horizontal paths and of non-trivial intervals (that is intervals not degenerate to a point). 
The tree of spaces-distance between any two points $x, y$ in $\tilde{X}$, denoted by $d_{\widetilde{X}}(x, y)$, is the infimum of the lengths of the paths in $\mathcal{P}(x, y)$, measured as the sum of the horizontal and interval-lengths of their subpaths.

This tree of spaces-distance is reminiscent of the quotient-metric of [5][§5.18]. The following lemma is obvious:

Lemma 2.11. With the notations of Definition 2.10, the space $\widetilde{X}$ equipped with the tree of spaces-distance $d_{\widetilde{X}}$ is a quasi geodesic metric space.

Remark 2.12. The geometric realization of a tree of geodesic spaces is the space we will work with. Thus, with a slight abuse of terminology we will often denote by $(\widetilde{X}, \pi, \mathcal{T})$ a tree of qi-embedded geodesic spaces and write "a tree of geodesic spaces ..." for "the geometric realization of a tree of geodesic spaces ...".

A section of a map $\pi: A \rightarrow B$ is a map $\sigma: B \rightarrow A$ such that $\pi \circ \sigma=\operatorname{Id}_{B}$ (this is only a set-theoretic notion, for instance we do not require that a section of a continuous map be continuous).

Definition 2.13. Let $(\widetilde{X}, \mathcal{T}, \pi)$ be the geometric realization of a tree of qi-embedded geodesic spaces.

For $v \geq 0$, a $v$-vertical segment in $\tilde{X}$ is a section $\sigma_{\omega}$ of $\pi$ over a geodesic $\omega$ of $\mathcal{T}$ which is a $(v+1, v)$-quasi isometric embedding of $\omega$ in $\left(\tilde{X}, d_{\tilde{X}}\right)$.

The vertical length of the $v$-vertical segment $\sigma_{\omega}: \omega \rightarrow \tilde{X}$ is the length $|\omega|_{\mathcal{T}}$.

We will not distinguish a vertical segment, which by definition is a map, from its image in the tree of spaces. Since a section is not necessarily continuous, this image is of course not a segment in the usual sense. But if $\omega=e_{i_{1}}^{\epsilon_{1}} \cdots e_{i_{k}}^{\epsilon_{k}}$ is a geodesic edge-path then a $v$-vertical segment over $\omega$ can be approximated by a sequence of intervals $x_{i} \times(0,1)$ over the $e_{i}$ 's, the Hausdorff distance between the $v$-vertical segment and these intervals only depending on $v$.

The "hallways-flare property" was introduced in [2]: it designated the main property introduced by the authors for the hyperbolicity of a graph of quasi isometrically embedded hyperbolic spaces. Our presentation here being very different and more dynamical in nature, we use the denomination of exponential-separation property for our central property given in Definition 2.14 below and invite the reader to compare with the "hallways-flare property" of [2].

Definition 2.14. (compare [2])

A tree of qi-embedded spaces satisfies the exponential-separation property if and only if for any $v \geq 0$ there exist $\lambda>1$ and positive integers $t_{0}, M$ such that, for any geodesic segment $[\beta, \gamma] \subset \mathcal{T}$ of length $2 t_{0}$ and midpoint $\alpha$, any two $v$-vertical segments $s_{0}, s_{1}$ over $[\beta, \gamma]$ with $d_{\text {hor }}\left(s_{0} \cap X_{\alpha}, s_{1} \cap X_{\alpha}\right) \geq M$ satisfy:

$$
\max \left(d_{\text {hor }}\left(s_{0} \cap X_{\beta}, s_{1} \cap X_{\beta}\right), d_{\text {hor }}\left(s_{0} \cap X_{\gamma}, s_{1} \cap X_{\gamma}\right)\right) \geq \lambda d_{\text {hor }}\left(s_{0} \cap X_{\alpha}, s_{1} \cap X_{\alpha}\right) .
$$

The constants $\lambda, M, t_{0}$ will be referred to as the constants of hyperbolicity.

We will sometimes say that the $v$-vertical segments are exponentially separated.

Remark 2.15. The exponential-separation property requires the exponential separation of the $v$-vertical segments for any $v \geq 0$. It suffices in fact that it be satisfied for some $v$ sufficiently large (see Lemma 3.7). 
Theorem 2.16. Let $\mathfrak{T}=\left(\mathcal{T},\left\{\left(X_{e}, \mathcal{P}_{e}\right)\right\},\left\{\left(X_{v}, \mathcal{P}_{v}\right)\right\},\left\{\jmath_{e}\right\}\right)$ be a tree of weakly relatively hyperbolic spaces. If $\widehat{\mathfrak{T}}$ satisfies the exponential-separation property then $\mathfrak{T}$ is weakly hyperbolic relative to the family composed of all the parabolic subspaces of the vertexspaces.

Remark 2.17. In the setting of weak relative hyperbolicity we could drop the assumption that the attaching-maps $\jmath_{e}$ be pair-maps from $\left(X_{e}, \mathcal{P}_{e}\right)$ to $\left(X_{t(e)}, \mathcal{P}_{t(e)}\right)$. In this case the statement of theorem 2.16 has to be modified by adding the collection of all the parabolic subspaces of the edge-spaces in the given family of parabolic subspaces for the tree of weakly relatively hyperbolic spaces. This follows from the proof of Theorem 2.16.

Definition 2.18. Let $\left(\mathcal{T},\left\{\left(X_{e}, \mathcal{P}_{e}\right)\right\},\left\{\left(X_{v}, \mathcal{P}_{v}\right)\right\},\left\{\jmath_{e}\right\}\right)$ be a tree of geodesic pairs.

The induced forest of parabolic spaces is the forest of spaces $\left(\mathcal{F}_{\mathcal{P}},\left\{\mathfrak{P}_{e}\right\},\left\{\mathfrak{P}_{v}\right\},\left\{\imath_{e}\right\}\right)$ defined as follows:

(a) There is a bijection $\sigma_{E}$ (resp. $\sigma_{V}$ ) from the set of edges (resp. vertices) of $\mathcal{F}_{\mathcal{P}}$ to the set of all the parabolic subspaces of the edge-spaces (resp. vertex-spaces) of $\mathcal{T}$.

(b) The edge-space $\mathfrak{P}_{e}\left(\right.$ resp. vertex-space $\mathfrak{P}_{v}$ ) of $\mathcal{F}_{\mathcal{P}}$ is the parabolic subspace $\sigma_{E}(e)$ (resp. parabolic subspace $\sigma_{V}(v)$ ) of $\mathcal{T}$.

(c) There is an oriented edge $e$ with terminal vertex $v$ in $\mathcal{F}_{\mathcal{P}}$ if and only if, letting $e^{\prime}$ be the oriented edge of $\mathcal{T}$ such that $\sigma_{E}(e) \subset X_{e^{\prime}}$ and $v^{\prime}$ the vertex of $\mathcal{T}$ such that $\sigma_{V}(v) \subset X_{v^{\prime}}$, one has $v^{\prime}=t\left(e^{\prime}\right)$ and $\jmath_{e^{\prime}}\left(\sigma_{E}(e)\right) \subset \sigma_{V}(v)$. In this case $\imath_{e}$ is the restriction of $\jmath_{e^{\prime}}$ to $\sigma_{E}(e)$.

An induced tree of parabolic spaces is any connected component of the induced forest of parabolic spaces.

Remark 2.19. The geometric realization of the induced forest of parabolic spaces of a tree of geodesic pairs is naturally embedded in the geometric realization of the latter. So, assimilating this forest and the tree to their geometric realizations, it makes sense to speak about the "horizontal distance between two induced trees of parabolic spaces" or about the vertical diameter of some of their subsets.

Definition 2.20. A tree of strongly relatively hyperbolic spaces satisfies the strong exponential-separation property if and only if it satisfies the exponential-separation property and for any $l \geq 0$ there is $t \geq 0$ such that for any two distinct induced trees of parabolic spaces, the union of all the strata where they are at horizontal distance smaller than $l$ has vertical diameter smaller than $t$.

Theorem 2.21. Let $\mathfrak{T}=\left(\mathcal{T},\left\{\left(X_{e}, \mathcal{P}_{e}\right)\right\},\left\{\left(X_{v}, \mathcal{P}_{v}\right)\right\},\left\{\jmath_{e}\right\}\right)$ be a tree of strongly relatively hyperbolic spaces. If $\widehat{\mathfrak{T}}$ satisfies the strong exponential-separation property then $\mathfrak{T}$ is strongly hyperbolic relatively to the family composed of all the induced trees of parabolic spaces.

2.1. Plan of the paper: The results above are consequences of Theorems 4.6 and 5.3 about the behavior of quasi geodesics in trees of hyperbolic spaces. Section 3 contains some technical consequences of the basic notions exposed above. Section 4 deals with the approximation of quasi geodesics in the particular case where all the attaching-maps of the considered tree of hyperbolic spaces are quasi isometries. Section 5 contains the adaptations to the general case. The important notions appearing in these two sections are the corridors in Section 4, and the generalized corridors in Section 5. These two sections appeal to two important Propositions whose proofs are delayed: Proposition 4.7 is proved in Section 8; Proposition 4.8 is proved in Section 9 whereas its adaptation to 
generalized corridors (Proposition 5.4) is dealt with in subsection 9.6. In Section 6 the reader will find the proof of Theorem 2.16 (weak relative hyperbolicity case) whereas Section 7 deals with the proof of Theorem 2.21 (strong relative hyperbolicity case). This last section also contains Proposition 7.4 whose proof is postponed to subsection 9.7.

\section{Preliminaries}

If $(X, d)$ is a metric space with distance function $d$, and $x$ a point in $X$, we set $B_{x}(r)=$ $\{y \in X ; d(x, y) \leq r\}$. If $A$ and $B$ are any two subsets of $(X, d), d(A, B)=\inf _{x \in A, y \in B} d(x, y)$. We set also $\mathcal{N}_{d}^{r}(A)=\{x \in X ; d(x, A) \leq r\}$ and $d^{H}(A, B)=\inf \{r \geq 0 ; A \subset$ $\mathcal{N}_{d}^{r}(B)$ and $\left.B \subset \mathcal{N}_{d}^{r}(A)\right\}$. The latter is the usual Hausdorff distance between $A$ and $B$. Finally, $\operatorname{diam}_{X}(A)$ stands for the diameter of $A$ : $\operatorname{diam}_{X}(A)=\sup \{d(x, y) ; x, y \in A\}$.

From now on, unless otherwise specified, the trees of qi-embedded spaces are equipped with the tree of spaces-distance $d_{\widetilde{X}}(.,$.$) introduced in Definition 2.10. This metric is a$ particular case of the telescopic metrics we define below. We recall that we also defined the horizontal distance, denoted by $d_{h o r}(.,$.$) , for pair of points belonging to a same stratum,$ and the horizontal length, denoted by $|.|_{\text {hor }}$ for horizontal paths, that is paths contained in a stratum. We adopt the convention that the horizontal distance is infinite for two points not belonging to a same stratum.

\subsection{The telescopic metric.}

Definition 3.1. Let $(\widetilde{X}, \mathcal{T}, \pi)$ be a tree of qi-embedded geodesic spaces, and let $v \geq 0$.

(a) A v-telescopic chain is an ordered sequence $\left(h_{0}, s_{0}, h_{1}, \cdots, h_{k-1}, s_{k-1}, h_{k}\right)$ of horizontal paths $h_{j}$ and of $v$-vertical segments $s_{j}$ in $\widetilde{X}$ such that:

- for any $k \geq j \geq 0, h_{j}$ belongs to a vertex-space,

- for any $k-1 \geq j \geq 0, t\left(h_{j}\right)=i\left(s_{j}\right)$ and $t\left(s_{j}\right)=i\left(h_{j+1}\right)$.

(b) The vertical length $|p|_{v e r t}^{v}$ of a $v$-telescopic chain $p=\left(h_{0}, s_{0}, h_{1}, \cdots, h_{k-1}, s_{k-1}, h_{k}\right)$ is equal to $\sum_{j=0}^{k-1}\left|s_{j}\right|_{\text {vert }}$.

(c) The horizontal length $|p|_{\text {hor }}^{v}$ of a $v$-telescopic chain $p=\left(h_{0}, s_{0}, h_{1}, \cdots, h_{k-1}, s_{k-1}, h_{k}\right)$ is equal to $\sum_{j=0}^{k}\left|h_{j}\right|_{h o r}$.

(d) The telescopic length $|p|_{\text {tel }}^{v}$ of a $v$-telescopic chain $p$ is equal to $|p|_{\text {hor }}^{v}+|p|_{\text {vert }}^{v}$.

(e) The $v$-telescopic distance $d_{\text {tel }}^{v}(x, y)$ between any two points $x$ and $y$ in $\tilde{X}$ is the infimum of the telescopic lengths of the $v$-telescopic chains between $x$ and $y$.

Remark 3.2. Setting $v=0$ in Definition 3.1 above we get the tree of spaces-distance (compare with Definition 2.10).

Let $v \geq 0$. The definition of a $v$-telescopic chain (item (a) of Definition 3.1) implies in particular that for any $k-1 \geq j \geq 0, \pi\left(s_{0}\right) \pi\left(s_{1}\right) \cdots \pi\left(s_{j}\right)$ is an edge-path between two vertices of $\mathcal{T}$. Any non-trivial (i.e. not degenerate to a point) $v$-vertical segment in a $v$-telescopic chain has $v$-vertical length greater or equal to one. For any $x \in \widetilde{X}$ there is $w \in V(\mathcal{T})$ such that $d_{\text {vert }}^{v}\left(x, \pi^{-1}(w)\right) \leq \frac{1}{2}$. It follows from the latter observation that, when dealing with the behavior of quasi geodesics or with the hyperbolicity of $\tilde{X}$, there is no harm in requiring that telescopic chains begin and end at strata over vertices of $\mathcal{T}$, as was done in Definition 3.1. 
For the sake of simplification, we will often forget the superscripts in the vertical, horizontal and telescopic lengths, unless some ambiguity might exist.

Lemma 3.3. Let $(\tilde{X}, \mathcal{T}, \pi)$ be a tree of hyperbolic spaces.

(a) For any $v \geq 0$ there exist $\lambda_{+} \equiv \lambda_{+}(v) \geq 1, \mu \equiv \mu(v) \geq 0$ such that, if $\omega_{0}$ and $\omega_{1}$ are any two $v$-vertical segments, with initial (resp. terminal) points $x_{0}, x_{1}$ (resp. $\left.y_{0}, y_{1}\right)$ and such that $\pi\left(\omega_{0}\right)=\pi\left(\omega_{1}\right)=[a, b]$ then:

$$
\frac{1}{\lambda_{+}^{d_{\mathcal{T}}(a, b)}} d_{\text {hor }}\left(x_{0}, x_{1}\right)-\mu \leq d_{h o r}\left(y_{0}, y_{1}\right) \leq \lambda_{+}^{d_{\mathcal{T}}(a, b)} d_{h o r}\left(x_{0}, x_{1}\right)+\mu
$$

The constants $\lambda_{+}, \mu$ will be referred to as the constants of quasi isometry.

(b) For any sequence of points $\left(x_{n}\right)_{n \in \mathbb{Z}^{+}}$in some stratum, $\lim _{n \rightarrow+\infty} d_{h o r}\left(x_{0}, x_{n}\right)=+\infty \Leftrightarrow$ $\lim _{n \rightarrow+\infty} d_{t e l}^{v}\left(x_{0}, x_{n}\right)=+\infty$

(c) For any $v, v^{\prime} \geq 0$, there exist $A \geq 1, B \geq 0$ such that the identity-map from $\left(\widetilde{X}, d_{t e l}^{v}\right)$ to $\left(\tilde{X}, d_{t e l}^{v^{\prime}}\right)$ is a $(A, B)$-quasi isometry.

(d) For any $d, v \geq 0$ there exists $C \equiv C(d, v) \geq 0$ such that for any $\alpha, \beta \in \mathcal{T}$ with $d_{\mathcal{T}}(\alpha, \beta)=d$, for any $x, y, z \in X_{\alpha}$ with $z \in[x, y]$, for any $x^{\prime}, y^{\prime}, z^{\prime} \in X_{\beta}$ which are the endpoints of $v$-vertical segments starting respectively at $x, y$ and $z$, we have $z^{\prime} \in \mathcal{N}_{\text {hor }}^{C}\left(\left[x^{\prime}, y^{\prime}\right]\right)$. Moreover, for any $d^{\prime} \geq d$ and $v^{\prime} \geq v, C\left(d^{\prime}, v^{\prime}\right) \geq C(d, v)$.

(e) For any $v, w \geq 0$, there is $D \geq 0$ such that if $s$ is a v-vertical segment, then $s$ is a $(D, D)$-quasi geodesic for the $w$-telescopic distance.

Proof. There is $\epsilon(v)$ such that each $\omega_{i}$ is at Hausdorff horizontal distance at most $\rho(v)$ of a vertical segment $\omega_{i}^{\prime}$ which is a sequence of intervals $x_{i}^{j} \times I$ over the edges $e_{j}$ in $[a, b]$, where $I=(0,1)$ for any $e_{j} \subset[a, b], I=(\epsilon, 1]$ if $e_{j} \cap[a, b]=\left[a, t\left(e_{j}\right)\right]$ and $I=(0, \epsilon]$ if $e_{j} \cap[a, b]=\left(i\left(e_{j}\right), b\right]$, with a jump of at most $\theta(v)$ with respect to the horizontal distance in each vertex-space intersected. More precisely, for any point $y$ in $[a, b], d_{h o r}\left(\omega_{i}(y), \omega_{i}^{\prime}(y)\right) \leq$ $\rho(v)$. Each edge-space is $(\mathfrak{a}, \mathfrak{b})$-quasi isometrically embedded into the vertex-spaces (see Definition 2.5). This implies for each edge $e_{j}$

$$
d_{\text {hor }}\left(\omega_{0}^{\prime} \cap X_{t\left(e_{j}\right)}, \omega_{1}^{\prime} \cap X_{t\left(e_{j}\right)}\right) \leq \mathfrak{a}\left(\mathfrak{a} d_{h o r}\left(\omega_{0}^{\prime} \cap X_{i\left(e_{j}\right)}, \omega_{1}^{\prime} \cap X_{i\left(e_{j}\right)}\right)+\mathfrak{b}\right)+\mathfrak{b}+2 \theta(v) .
$$

Since the $v$-vertical segments $\omega_{i}^{\prime}$ are at horizontal distance smaller than $\rho(v)$ from the $\omega_{i}$ we get

$$
d_{h o r}\left(\omega_{0} \cap X_{t\left(e_{j}\right)}, \omega_{1} \cap X_{t\left(e_{j}\right)}\right) \leq \mathfrak{a}\left(\mathfrak{a} d_{h o r}\left(\omega_{0} \cap X_{i\left(e_{j}\right)}, \omega_{1} \cap X_{i\left(e_{j}\right)}\right)+\mathfrak{b}\right)+\mathfrak{b}+2 \theta(v)+4 \rho(v) .
$$

Item (a) follows by composing the inequalities given by all the edges: it suffices to set $\lambda_{+}=\mathfrak{a}^{2}+1$ and $\mu=\left(\mathfrak{a}^{2}+1\right)(\mathfrak{a} \mathfrak{b}+\mathfrak{b}+2 \theta(v)+4 \rho(v))$.

If $d_{t e l}^{v}\left(x_{0}, x_{n}\right)$ does not tend toward infinity with $n$, then both the horizontal lengths and the vertical length of the quasi geodesic telescopic chains between $x_{0}$ and $x_{n}$ are bounded above by some constant $M$. Item (a) then gives $\lambda_{+}(v)$ and $\mu(v)$ such that $d_{h o r}\left(x_{0}, x_{n}\right)$ is bounded above by $\lambda_{+}^{M}(v)(M+\mu(v))$. Conversely, if $d_{h o r}\left(x_{0}, x_{n}\right)$ does not tend toward infinity with $n$ then neither does $d_{t e l}^{v}\left(x_{0}, x_{n}\right)$ since horizontal geodesics are $v$-telescopic chains. We so proved item (b).

To prove item (c), it is sufficient to check that for any $v \geq 0$, the identity-map is a quasi isometric embedding from $\left(\widetilde{X}, d_{\tilde{X}}\right)$ to $\left(\widetilde{X}, d_{t e l}^{v}\right)$. For this sake, just observe that there is some constant $X(v)$ such that the $v$-telescopic length of a 0-telescopic chain $g$ of $\left(\widetilde{X}, d_{\tilde{X}}\right)$ is bounded above by $X(v)$ times the length of $g$ plus $X(v)$. Conversely, there are constants $Y(v) \geq 1$ and $Z(v) \geq 0$ such that any $v$-telescopic chain $g$ is at Hausdorff 
distance smaller than $Z(v)$ from a 0 -telescopic chain whose length is bounded above by $Y(v)$ times the $v$-telescopic length of $g$ plus $Y(v)$. From the associated inequalities, we get that the identity-map is a quasi-isometric embedding from one space to the other, so that item (c) is proved since the identity is surjective.

Item (d) amounts to saying that the image of a geodesic under a $(a, b)$-quasi isometric embedding is $C(a, b)$-close to any geodesic between the images of the endpoints. This is a well-known assertion, see for instance [7].

To prove item (e) observe that by the definition a $v$-vertical segment is a geodesic between its endpoints for the $v$-telescopic distance. Item (e) then follows from item (c).

Remark 3.4. Throughout the remainder of this text, the constants appearing in each lemma, corollary or proposition will be denoted by $C, D, \cdots$ and thereafter they will be referred to by the same letter with the number of the lemma, corollary or proposition in subscript. For instance, if Lemma 3.4 introduces the constants $C$ and $D$ we will refer to these constants as $C_{3.4}$ and $D_{3.4}$.

3.2. Exponential separation. A subset $S$ of a Gromov hyperbolic space $X$ is quasi convex if there exists a constant $C$ such that any geodesic (quasi geodesic, with the constant $C$ then depending on the constants of "quasi geodesicity", in the case where $X$ is a quasi geodesic space) between any two points in $S$ is contained in the $C$-neighborhood of $S$.

Definition 3.5. Let $\widetilde{X}$ be a tree of hyperbolic spaces and let $S$ be a horizontal subset which is quasi convex in its stratum, for the horizontal metric. If $x$ is any point in $\widetilde{X}$ then a horizontal quasi projection of $x$ to $S$, denoted by $P_{S}^{\text {hor }}(x)$, is any point $y$ in $S$ such that $d_{\text {hor }}(x, y) \leq d_{\text {hor }}(x, S)+1$.

If $x$ and $S$ do not belong to a same stratum, such a horizontal quasi projection does not exist, the horizontal distance $d_{\text {hor }}(x, y)$ being infinite for any $y \in S$.

Lemma 3.6. Let $\delta \geq 0$ and let $\left(\mathcal{T},\left\{X_{e}\right\},\left\{X_{v}\right\},\left\{\jmath_{e}\right\}\right)$ be a tree of $\delta$-hyperbolic spaces.

(a) For any $t \geq 0$ there exists $C(t) \geq 0$, increasing with $t$, such that if $v \geq C(t)$, if $p=e_{1} \cdots e_{n}$ is a reduced edge-path of $\mathcal{T}$ with $n \leq t+1$ such that the map $\jmath_{p}=\jmath_{e_{n}} \circ \jmath_{e_{n}^{-1}} \circ \cdots \jmath_{e_{1}} \circ \jmath_{e_{1}^{-1}}$ is defined over some non-empty subset of $X_{i\left(e_{1}\right)}$, if $h$ is contained in the horizontal $2 \delta$-neighborhood of some horizontal geodesic between two points in $\jmath_{p}\left(X_{e_{n}}\right)$ then there are v-vertical segments over $p$ starting at any point of $h$.

We set $C \equiv C(1)$.

(b) There is $D \geq 0$ such that for any $v \geq C$, if $e$ is an edge of $\mathcal{T}$ and $h$ is a horizontal geodesic in $X_{t(e)}$ such that no v-vertical segment starting at $h$ can be defined over $e$, then

$$
\operatorname{diam}_{X_{t(e)}}\left(P_{h}^{h o r}\left(\jmath_{e}\left(X_{e}\right)\right)\right) \leq D
$$

(c) For any $v \geq C$, if for any edge e of $\mathcal{T}$ the map $\jmath_{e}$ is a $(\mathfrak{a}, \mathfrak{b})$-quasi isometry (and not only a $(\mathfrak{a}, \mathfrak{b})$-quasi isometric embedding - see Definition 2.5) then for any reduced edge-path $p$ in $\mathcal{T}$, for any point $x$ in any stratum over any point in $p$, there is a $v$-vertical segment over $p$ through $x$.

Proof of Lemma 3.6. By definition of a tree of $\delta$-hyperbolic spaces, each stratum is $\delta$ hyperbolic for the horizontal metric. Moreover the map $\jmath_{p}$ is a quasi isometric embedding with constants of quasi isometry depending on $n$ (the length of the edge-path - see item (a) of Lemma 3.3) . This gives a constant $c$ depending on $n$ such that for any two points 
$x, y \in \jmath_{e}\left(X_{e}\right)$, any horizontal geodesic $[x, y]$ lies in the horizontal $c$-neighborhood of the image of $\jmath_{p}$. Of course, any point $x \in X_{t(p)}$ in this image is the endpoint of a 0-vertical segment over $p$ : it suffices to map $t(p)$ to $x$ and, if we denote by $x_{i}$ the pre-image of $x$ in $X_{t\left(e_{i}\right)}$ (the $e_{i}$ are the edges of $p$ ), choose the isometric embedding of each $e_{i}$ into $\widetilde{X}$ to be the interval $\left[x_{i}, x_{i+1}\right]$. Since $h$ is contained in the horizontal $2 \delta$-neighborhood of some horizontal geodesic between two points in the image of $\jmath_{p}$ then any point in $h$ is at horizontal distance at most $2 \delta+c$ of some point in this image and therefore of the endpoint of a 0 -vertical segment over $p$. Thus any point in $h$ is the initial point of some $v$-vertical segment over $p$ as soon as $v$ is chosen sufficiently large with respect to $2 \delta+c$ (recall that $c$ depends on $n$ ). We so get item (a).

Let us prove item (b). Let $h$ be a horizontal geodesic. If there exist $x, y$ in the image of $\jmath_{e}$ such that $[x, y] \cap \mathcal{N}_{h o r}^{2 \delta}(h) \neq \emptyset$, then it was proved above that, if $v \geq C(1)$, there is a point in $h$ which is the initial point of a $v$-vertical segment over $e$. Since it is assumed that no such $v$-vertical segment exists, we get $[x, y] \cap \mathcal{N}_{\text {hor }}^{2 \delta}(h)=\emptyset$ for any $x, y$ in the image. By the $2 \delta$-thiness of the geodesic rectangles, it follows that $\operatorname{diam}_{X_{t(e)}}\left(P_{h}^{h o r}\left(\jmath_{e}\left(X_{e}\right)\right)\right) \leq D$ for some constant $D$ depending only on $\delta$. We so got item (b).

Finally, since each map $\jmath_{e}$ is a $(\mathfrak{a}, \mathfrak{b})$-quasi isometry, by definition, any point in any vertex-space is at horizontal distance at most $\mathfrak{b}$ from the endpoint of a 0 -vertical segment over any edge of $\mathcal{T}$ incident to this vertex. The concatenation of any arbitrary number of 0 -vertical segments whose endpoints are at horizontal distance at most $\mathfrak{b}$ one from the other yields a $(\mathfrak{b}+1,(\mathfrak{b}+1) \mathfrak{b})$-vertical segment. Hence the conclusion.

See Definition 2.14 for the constants of hyperbolicity referred to in Lemma 3.7 below.

Lemma 3.7. Let $(\tilde{X}, \mathcal{T}, \pi)$ be a tree of hyperbolic spaces. Assume that the v-vertical segments of $\tilde{X}$ are exponentially separated with constants of hyperbolicity $\lambda_{v}>1, M_{v}, t_{0} \geq$ 0 . If $v \geq C_{3.6}\left(t_{0}\right)$ then for any $w \geq 0$, the $w$-vertical segments are exponentially separated, with constants of hyperbolicity $\lambda_{w}>1, M_{w} \geq 0$ and $t_{0}$.

Proof. The statement is a tautology if $w \leq v$. We thus assume $w \geq v$. Consider $\alpha, \beta, \gamma$ in $\mathcal{T}$ with $\alpha \in[\beta, \gamma]$ and $d_{\mathcal{T}}(\alpha, \beta)=d_{\mathcal{T}}(\alpha, \gamma)=t_{0}$. Consider two $w$-vertical segments $S_{0}, S_{1}$ over $[\beta, \gamma]$ with $d_{\text {hor }}\left(x_{0}, x_{1}\right) \geq M$, where $x_{i}=S_{i} \cap X_{\alpha}$ and $M>M_{v}$. We distinguish two cases:

Case 1: There exist v-vertical segments $s_{0}, s_{1}$ passing through $x_{0}, x_{1}$ and defined over $[\beta, \gamma]$. From item (a) of Lemma 3.3, each endpoint of the $s_{i}$ 's is at bounded horizontal distance $c$ from an endpoint of a $S_{i}$, where the upper-bound only depends on $w, t_{0}$ and the constants of quasi isometry. Thus choosing $M_{w}$ sufficiently large with respect to $w$ so that there exists $1<\lambda_{w} \leq \lambda-2 \frac{c}{M_{w}}$ gives the inequality of exponential separation between $d_{\text {hor }}\left(x_{0}, x_{1}\right)$ and $\max \left(d_{\text {hor }}\left(S_{0} \cap X_{\beta}, S_{1} \cap X_{\beta}\right), d_{\text {hor }}\left(S_{0} \cap X_{\gamma}, S_{1} \cap X_{\gamma}\right)\right)$.

Case 2: There exists no v-vertical segments passing through $x_{0}, x_{1}$ and defined over $[\beta, \gamma]$. Since $w \geq v \geq C_{3.6}\left(t_{0}\right)$, if we choose $M_{w}$ sufficiently large, then there is a subgeodesic $h_{0} \subset\left[x_{0}, x_{1}\right]$ which is in the $2 \delta$-neighborhood of some horizontal geodesic between two points in $\jmath_{[\beta, \alpha]}\left(X_{\beta}\right)$ and also of some horizontal geodesic between two points in $\jmath_{[\gamma, \alpha]}\left(X_{\gamma}\right)$. For a given $w$, the horizontal length of $h_{0}$ increases with $M_{w}$ since it is assumed that $w$-vertical segments pass through $x_{0}$ and $x_{1}$. More precisely, $\frac{\left|h_{0}\right|_{h o r}}{d_{h o r}\left(x_{0}, x_{1}\right)}$ tends toward 1. Indeed, by Lemma 3.3 item (b), the telescopic distance from $x_{0}, x_{1}$ to the embedded edge-spaces "increases with the corresponding horizontal distances", so that a too large horizontal distance from $x_{0}, x_{1}$ to the embedded edge-spaces would forbid the existence of $w$-vertical segment over $[\beta, \gamma]$ through $x_{0}$ and $x_{1}$. 
Moreover, since $v \geq C_{3.6}\left(t_{0}\right)$, by Lemma 3.6 item (a) there are $v$-vertical segments passing through the endpoints of $h_{0}$ defined over $[\beta, \gamma]$. Thus in a first step, since the exponential separation holds for $v$-vertical segments through the endpoints of $h_{0}$ and we can make $\left|h_{0}\right|_{\text {hor }}$ as large as we wish by increasing $M_{w}$, by the same computations as in the first case, we take $M_{w}$ sufficiently large so that the exponential separation holds for vertical $w$-segments through the endpoints of $h_{0}$. Then using that $\frac{\left|h_{0}\right|_{h o r}}{d_{h o r}\left(x_{0}, x_{1}\right)}$ approaches 1 when increasing $M_{w}$, we get that the exponential separation for $w$-vertical segments through the endpoints of $h_{0}$ implies the exponential separation for $w$-vertical segments through $x_{0}$ and $x_{1}$.

We end this section with a simple lemma about the constants of hyperbolicity.

Lemma 3.8. Let $(\widetilde{X}, \mathcal{T})$ be a tree of hyperbolic spaces satisfying the exponential-separation property.

(a) The constants of hyperbolicity and quasi isometry can be chosen arbitrarily large.

(b) For any constants of hyperbolicity $\lambda, M, t_{0}$ such that $M$ is sufficiently large, there exists $C \geq 0$ such that the following holds:

For any $\alpha \in \mathcal{T}$, for any $\beta$ with $d_{\mathcal{T}}(\alpha, \beta)=t_{0}$, for any two $v$-vertical segments $s_{0}, s_{1}$ over $[\beta, \alpha]$ such that $d_{\text {hor }}\left(s_{0} \cap X_{\alpha}, s_{1} \cap X_{\alpha}\right) \geq M$, if

$$
\frac{1}{\lambda} d_{h o r}\left(s_{0} \cap X_{\alpha}, s_{1} \cap X_{\alpha}\right)<d_{h o r}\left(s_{0} \cap X_{\beta}, s_{1} \cap X_{\beta}\right),
$$

then, for any $n \geq 1$, for any $\mathcal{T}$-geodesic $\omega$ starting at $\alpha$ with $[\alpha, \beta] \subset \omega$ and $|\omega|_{\mathcal{T}} \geq C+n t_{0}:$

$$
d_{\text {hor }}\left(\omega\left(s_{0} \cap X_{\alpha}\right), \omega\left(s_{1} \cap X_{\alpha}\right)\right) \geq \lambda^{n} d_{h o r}\left(s_{0} \cap X_{\alpha}, s_{1} \cap X_{\alpha}\right),
$$

where the notation $\omega x$ denotes the set of points $y \in \widetilde{X}$ such that some v-vertical segment $s$ with $\pi(s)=\omega$ connects $x$ to $y$.

Proof. Item (a) is obvious. We just give a sketch of a proof of item (b) and leave the remaining details to the reader. Observe that the assumption of item (b) tells us that the horizontal distance between the points $s_{0} \cap X_{\alpha}$ and $s_{1} \cap X_{\alpha}$ is not exponentially contracted with factor $1 / \lambda$ in $X_{\beta}$, or equivalently the points $s_{0} \cap X_{\beta}$ and $s_{1} \cap X_{\beta}$ are not exponentially separated in $X_{\alpha}$. Thus they are exponentially separated in the other directions: this is the idea developed below.

By the exponential separation property, the assumption $\frac{1}{\lambda} d_{\text {hor }}\left(s_{0} \cap X_{\alpha}, s_{1} \cap X_{\alpha}\right)<$ $d_{\text {hor }}\left(s_{0} \cap X_{\beta}, s_{1} \cap X_{\beta}\right)$ implies that the points $s_{0} \cap X_{\beta}, s_{1} \cap X_{\beta}$ are exponentially separated in every direction at $\beta$ which does not intersect $[\alpha, \beta)$. Now, there is a constant $D(v)$ such that, when considering other $v$-vertical segments $s_{0}^{\prime}, s_{1}^{\prime}$ starting respectively at $s_{0} \cap X_{\alpha}$ and $s_{1} \cap X_{\alpha}, d_{h o r}\left(s_{i}^{\prime}, s_{i}\right) \leq D(v)$. Thus, taking $M$ and $C$ sufficiently large, we get that the multiplication by $\lambda^{E\left[C / t_{0}\right]}$ (where $E[$.] is the integer part) between $\alpha$ and $\zeta$ with $d_{\mathcal{T}}(\zeta, \alpha)=C$ and $\beta \in[\alpha, \zeta]$ is sufficiently large to compensate the horizontal deviation, which only consists of summing constants $D(v)$, caused by the fact that $v$ vertical segments over a same geodesic of $\mathcal{T}$ do not necessarily end at the same points: we so get, when $C$ is sufficiently large, a multiplication by $\lambda$ of $d_{h o r}\left(s_{0} \cap X_{\alpha}, s_{1} \cap X_{\alpha}\right)$. The exponential separation property then implies that for each $n t_{0}$ thereafter one has a multiplication by $\lambda^{n}$. 


\section{Approximation of QUASi Geodesics: A "Simple" CASE}

The corridors (and later the generalized corridors) defined below are not the hallways of [2]. The reason is that we are interested in exhibiting quasi convex subsets of our trees of hyperbolic spaces and the hallways of [2], in general, are not quasi convex.

Definition 4.1. Let $(\widetilde{X}, \mathcal{T}, \pi)$ be a tree of hyperbolic spaces, and let $v \geq 0$.

A $v$-vertical tree is a $(v+1, v)$-quasi isometric embedding $\sigma: T \rightarrow \widetilde{X}$ of a subtree $T$ of $\mathcal{T}$ into $\left(\tilde{X}, d_{\tilde{X}}\right)$ which is a section of $\pi$.

A $v$-vertical tree $\sigma: T \rightarrow \widetilde{X}$ is maximal if and only if there exists no $v$-vertical tree $\sigma^{\prime}: T^{\prime} \rightarrow \widetilde{X}$ such that $T \subset T^{\prime}, T \neq T^{\prime}$ and $\sigma_{\mid T}^{\prime}=\sigma$.

A $v$-corridor $\mathcal{C}$ is a subset of $\widetilde{X}$ for which there exist two maximal $v$-vertical trees $\sigma_{i}: T_{i} \rightarrow \widetilde{X}(i=1,2)$ termed the vertical boundaries of $\mathcal{C}$, with the following properties:

(a) If $T=T_{1} \cap T_{2}$ then for each $\alpha \in T, \mathcal{C} \cap X_{\alpha}$ is a horizontal geodesic with endpoints $\sigma_{1}\left(T_{1}\right) \cap X_{\alpha}$ and $\sigma_{2}\left(T_{2}\right) \cap X_{\alpha}$.

The subtree $T$ of $\mathcal{T}$ is the core of $\mathcal{C}$ and the union of the horizontal geodesics in the strata over the ends of $T$, if any, is the horizontal boundary of $\mathcal{C}$.

(b) For any $\alpha \in T_{i} \backslash T, \mathcal{C} \cap X_{\alpha}=\sigma_{i}(\alpha)$.

(c) For any $\alpha \notin T_{1} \cup T_{2}, \mathcal{C} \cap X_{\alpha}=\emptyset$.

Remark 4.2. Let $(\widetilde{X}, \mathcal{T}, \pi)$ be a tree of hyperbolic spaces the attaching-maps of which are all quasi isometries (and not only quasi isometric embeddings). Then, from item (c) of Lemma 3.6, as soon as $v \geq C_{3.6}$, given any two points $x, y$ in $\widetilde{X}$ there is a $v$-corridor $\mathcal{C}$ with $\operatorname{Core}(\mathcal{C})=\mathcal{T}$, whose vertical boundaries $\sigma_{i}: \mathcal{T} \rightarrow \widetilde{X}$ pass through $x$ and $y$.

Definition 4.3. Let $\mathcal{C}$ be a union of horizontal geodesics in a tree of hyperbolic spaces $(\widetilde{X}, \mathcal{T})$. Assume that for each stratum $X_{\alpha}$ the intersection $\mathcal{C} \cap X_{\alpha}$ is either empty or a horizontal geodesic.

If $x$ is a point in a stratum $X_{\alpha}$ such that $\mathcal{C} \cap X_{\alpha}$ is non-empty, then $P_{\mathcal{C}}^{\text {hor }}(x)$ stands for the horizontal quasi projection $P_{\mathcal{C} \cap X_{\alpha}}^{h o r}(x)$ of $x$ to $\mathcal{C}$ (see Definition 3.5).

In the definition above, for instance $\mathcal{C}$ might be a corridor. Before stating Lemma 4.4 below, we would like to insist on the fact that the horizontal quasi projection $P_{\mathcal{C}}^{\text {hor }}$ is a projection in the strata which only refers to the horizontal metric defined on each stratum.

Lemma 4.4. Let $(\tilde{X}, \mathcal{T}, \pi)$ be a tree of hyperbolic spaces.

For any $v \geq C_{3.6}$, there exists $C \equiv C(v) \geq v$ such that, if $\mathcal{C}$ is a v-corridor in $\tilde{X}$ then for any v-vertical segment $s$ with $\pi(s) \subset \pi(\overline{\mathcal{C}}), P_{\mathcal{C}}^{\text {hor }}(s)$ is a $C$-vertical segment.

In particular for any $v$-corridor $\mathcal{C}$ in $\tilde{X}$, the $C(v)$-telescopic distance $d_{\text {tel }}^{C(v)}: \mathcal{C} \times \mathcal{C} \rightarrow$ $\mathbb{R}^{+}$, which is the infimum of the lengths of the $C(v)$-telescopic chains in $\mathcal{C}$ between the considered points, is well-defined and $\left(\mathcal{C}, d_{\text {tel }}^{C(v)}\right)$ is a quasi geodesic metric space.

Figure 1 illustrates Lemma 4.4.

Proof. If $\sigma: \omega \rightarrow \widetilde{X}$ is the section of $\pi$ such that $s=\sigma(\omega)$ then $P_{\mathcal{C}}^{\text {hor }}(s)$ is the image of $\omega$ under the map $P_{\mathcal{C}}^{h o r} \circ \sigma$. This map is a section of $\pi$ since the horizontal quasi projection $P_{\mathcal{C}}^{\text {hor }}$ is a projection in each stratum. We want to prove the existence of $C(v)$ independent of $\omega$ such that $P_{\mathcal{C}}^{h o r} \circ \sigma$ is a $(C(v)+1, C(v))$-quasi isometric embedding of $\omega$ into $\widetilde{X}$. Assume that $\omega$ is (contained in) a single edge. Let $z=\left(P_{\mathcal{C}}^{\text {hor }} \circ \sigma\right)(i(\omega))$. Since $v \geq C_{3.6}$ and since 


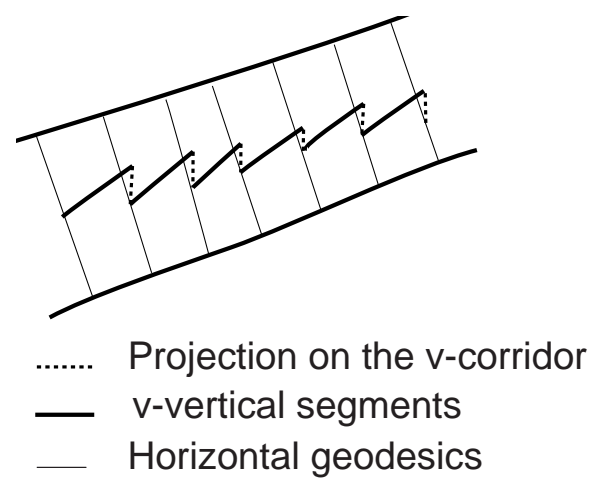

FiguRE 1.

$\mathcal{C}$ is a $v$-corridor, if it is defined over $\omega$, by item (a) of Lemma $3.6 v$-vertical segments can be defined over $\omega$ starting at $z$. By item (d) of Lemma 3.3, the endpoint $z^{\prime}$ of a $v$ vertical segment starting at $z$ is in the horizontal $C_{3.3}(1, v)$-neighborhood of $\mathcal{C}$. Consider a horizontal geodesic rectangle with vertices $z^{\prime}, \sigma(t(\omega)), P_{\mathcal{C}}^{\text {hor }}\left(z^{\prime}\right)$ and $\left(P_{\mathcal{C}}^{\text {hor }} \circ \sigma\right)(t(\omega))$. By the $\delta$-hyperbolicity of the strata, it is $2 \delta$-thin. From item (a) of Lemma 3.3 , the length of the subgeodesic of $\left[z^{\prime}, \sigma(t(\omega))\right]$ which is in the horizontal $2 \delta$-neighborhood of $\left[P_{\mathcal{C}}^{h o r}\left(z^{\prime}\right),\left(P_{\mathcal{C}}^{h o r} \circ \sigma\right)(t(\omega))\right]$ is bounded above by some constant only depending on $\delta$ and on the constants of quasi isometry embeddings for $v$-vertical segments. Thus the point $z^{\prime}$ is at bounded horizontal distance from $\left(P_{\mathcal{C}}^{\text {hor }} \circ \sigma\right)(t(\omega))$, the bound being independent from the chosen $v$-vertical segment $s$ and edge $\omega$ of $\mathcal{T}$. We so easily get that $P_{\mathcal{C}}^{\text {hor }} \circ \sigma$ is a $(d(v), d(v)+1)$-quasi isometric embedding for some constant $d(v)$ if the image of $\pi \circ \sigma$ is (contained in) an edge of $\mathcal{T}$. As in the proof of item (c) of Lemma 3.6, a concatenation of $d(v)$-vertical segments $s_{i}$ such that the terminal point of $s_{i}$ is the initial point of $s_{i+1}$ defines a $C(v)$-vertical segment for some constant $C(v)$. This proves the first - and main - assertion of the current lemma. It readily follows that any two points $x, y \in \mathcal{C}$ are connected by a $C(v)$-telescopic chain in $\mathcal{C}$ so that the distance $d_{t e l}^{C(v)}(x, y)$ is never infinite (and of course never zero if $x \neq y$ ). The $C(v)$-telescopic metric on $\mathcal{C}$ is then well-defined. It makes $\mathcal{C}$ a quasi geodesic space in the same way as the telescopic distance makes $\widetilde{X}$ a quasi geodesic space.

Definition 4.5. Let $(\widetilde{X}, \mathcal{T}, \pi)$ be a tree of qi-embedded geodesic spaces, let $v \geq 0$.

(a) The diagonal distance between two maximal $v$-vertical trees $\sigma_{i}: T_{i} \rightarrow \widetilde{X}$ is the infimum of the horizontal lengths of the horizontal geodesics between $\sigma_{1}\left(T_{1}\right)$ and $\sigma_{2}\left(T_{2}\right)$. The diagonal distance is infinite if no such horizontal geodesic exists.

(b) A diagonal between two maximal v-vertical trees $\sigma_{i}: T_{i} \rightarrow \widetilde{X}$ is any horizontal geodesic $D$ between $\sigma_{1}\left(T_{1}\right)$ and $\sigma_{2}\left(T_{2}\right)$ which is contained in some vertex-space, and whose horizontal length is less or equal to the diagonal distance plus 1 .

(c) A diagonal is a horizontal geodesic $D$ for which there exist two maximal $v$-vertical trees $\sigma_{i}: T_{i} \rightarrow \widetilde{X}$ passing through its endpoints such that $D$ is a diagonal between $\sigma_{1}\left(T_{1}\right)$ and $\sigma_{2}\left(T_{2}\right)$.

See Figure 2. The diagonal distance between two vertical trees may vanish (hence the diagonal distance is in fact a pseudo-distance). A diagonal may be reduced to a single point.

Before the statement of Theorem 4.6, we would like to point out that this is a theorem about trees of hyperbolic spaces whose attaching-maps are quasi isometries, and not 


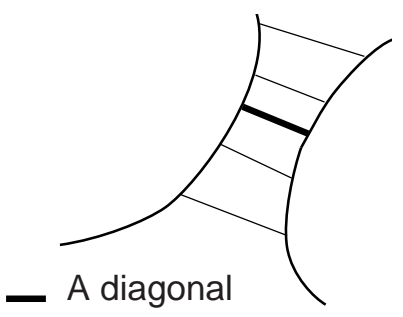

FIGURE 2.

only quasi isometric embeddings. This simplification has an important consequence, see Remark 4.2. The main feature of this theorem is to approximate quasi geodesics of $\widetilde{X}$ by "canonical" quasi geodesics.

Theorem 4.6. Let $\widetilde{X}$ be a tree of hyperbolic spaces which satisfies the exponentialseparation property. Assume that each attaching-map from an edge-space into a vertexspace is a quasi isometry. Then for any $v \geq C_{3.6}$, for any $L$ greater than some critical constant, for any $a \geq 1$ and $b \geq 0$, there are $E \equiv E(v) \geq v, D \equiv D(L, v) \geq L$, $C \equiv C(L, v, a, b) \geq 0$, such that the following holds:

For any $v$-corridor $\mathcal{C}$ whose vertical boundaries $B_{0}$ and $B_{1}$ are at diagonal distance at least $D$, there is an E-telescopic chain $\mathcal{P}=\left(h_{0}, s_{0}, h_{1}, \cdots, s_{k-1}, h_{k}\right)$ in $\mathcal{C}$ satisfying the following properties:

(a) $\mathcal{P}$ is a $(D, D)$-quasi geodesic of $\left(\widetilde{X}, d_{\tilde{X}}\right)$ which connects $B_{0}$ to $B_{1}$.

(b) For any $0 \leq i \leq k-1, h_{i}$ is a length $L$ diagonal and $\left|h_{k}\right|_{\text {hor }} \leq D$.

(c) For any $(a, b)$-quasi geodesic $g$ in $\widetilde{X}$ with endpoints in $B_{0}$ and $B_{1}$, if $t_{i} \subset B_{i}$ is the $v$-vertical segment in $B_{i}$ from $\mathcal{P}$ to $g$ then $\left(*, t_{0}^{-1}, \mathcal{P}, t_{1}, *\right)$, where $*$ denotes the trivial (i.e. degenerate to a point) horizontal path, defines a $(D, D)$-quasi geodesic E-telescopic chain whose Hausdorff distance from $g$ in $\left(\widetilde{X}, d_{\tilde{X}}\right)$ is bounded above by $C$.

If $\mathcal{C}$ is a $v$-corridor whose vertical boundary trees $B_{0}, B_{1}$ are at diagonal distance smaller than $D$ then any $(a, b)$-quasi geodesic $g$ in $\widetilde{X}$ with endpoints in $B_{0}$ and $B_{1}$ is contained in the telescopic $C$-neighborhood of $B_{0} \cup B_{1}$. More precisely, $g$ is at Hausdorff distance smaller than $C$ from a telescopic chain of the form $\left(*, t_{0}^{-1}, h_{0}, t_{1}, *\right)$ where $h_{0}$ is a (possibly degenerate to a point) horizontal geodesic in $\mathcal{C}$ with horizontal length smaller than $D$, and $t_{i}$ is the v-vertical segment in $B_{i}$ from $h_{0}$ to $g$.

For proving this theorem, we need two important propositions which we state now but whose proofs are postponed until Sections 8 and 9. For the understanding of Proposition 4.7 , let us recall that we proved in Lemma 4.4 that the $w$-telescopic metric is well-defined over any $v$-corridor $\mathcal{C}$ in a tree of hyperbolic spaces $\widetilde{X}$ as soon as $w$ is sufficiently large. The corridor $\mathcal{C}$ then becomes a quasi geodesic metric space when equipped with this telescopic metric and this quasi geodesic metric space is denoted by $\left(\mathcal{C}, d_{\text {tel }}^{w}\right)$.

Proposition 4.7. Let $\widetilde{X}$ be a tree of hyperbolic spaces which satisfies the exponentialseparation property.

For any $v \geq C_{3.6}$, there is $D \geq v$ such that for any $w \geq D, L>0, a \geq 1$ and $b \geq 0$ there exists $C \geq 0$ such that if $\mathcal{C}$ is a v-corridor in $\widetilde{X}$, if $L$ is the horizontal length of some horizontal geodesic $[x, y]$ in $\mathcal{C}$, if $g$ is a $(a, b)$-quasi geodesic of $\left(\mathcal{C}, d_{\text {tel }}^{w}\right)$ from a $w$ vertical tree through $x$ in $\mathcal{C}$ to a w-vertical tree through $y$ in $\mathcal{C}$, then $g$ is contained in the $C$-neighborhood of the union of the $w$-vertical segments connecting its endpoints to $x$ 
and $y$. If $L$ is greater than some critical constant then for any $L^{\prime}>L^{\prime \prime} \geq L$, we have $C\left(L^{\prime}\right)>C\left(L^{\prime \prime}\right) \geq C(L)$.

See Section 8 for a proof, and Figure 3 for an illustration.

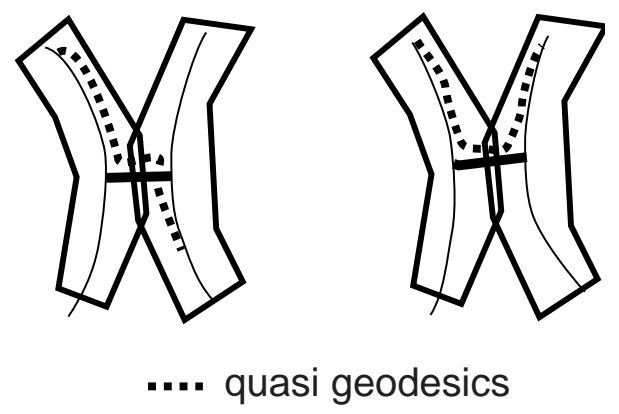

FIGURE 3.

Proposition 4.8. Let $\widetilde{X}$ be a tree of hyperbolic spaces which satisfies the exponentialseparation property and the attaching-maps of which are quasi isometries.

For any $v \geq C_{3.6}, a \geq 1$ and $b \geq 0$ there exists $C \geq 0$ such that, if $g$ is a $(a, b)$ quasi geodesic in $\widetilde{X}$ and if $\mathcal{C}$ is a v-corridor whose vertical boundaries pass through the endpoints of $g$ then

$$
g \subset \mathcal{N}_{\widetilde{X}}^{C}(\mathcal{C})
$$

where $\mathcal{N}_{\widetilde{X}}^{C}(\mathcal{C})$ denotes the $C$-neighborhood of $\mathcal{C}$ for the telescopic metric.

See Section 9 for a proof.

We will also need the following two much easier statements.

Lemma 4.9. Let $\widetilde{X}$ be a tree of hyperbolic spaces.

There exists $C \geq 0$ such that for any $v \geq 0$, for any $v$-corridor $\mathcal{C}$ in $\tilde{X}$, for any two points $x, y$ in a same stratum intersected by $\mathcal{C}, d_{\text {hor }}\left(P_{\mathcal{C}}^{\text {hor }}(x), P_{\mathcal{C}}^{\text {hor }}(y)\right) \leq d_{\text {hor }}(x, y)+C$. The same inequality holds for the horizontal quasi projections of $x$ and $y$ to the image of the embedding of an edge-space into a vertex-space.

Proof. Since there is $\delta \geq 0$ such that strata are $\delta$-hyperbolic spaces for the horizontal metric and the subspaces to which one projects are quasi convex subsets of their stratum for this horizontal metric, this is a consequence of [7], Corollary 2.2.

Lemma 4.10. Let $\widetilde{X}$ be a tree of hyperbolic spaces.

For any $v \geq C_{3.6}, a \geq 1$ and $b, r \geq 0$ there are $C \equiv C(v, a, b, r) \geq 1$ and $D \equiv D(v)$ such that for any $v$-telescopic $(a, b)$-quasi geodesic $g$ of $\widetilde{X}$ and for any $v$-corridor $\mathcal{C}$, if $g \subset \mathcal{N}_{\text {hor }}^{r}(\mathcal{C})$ then any horizontal quasi projection $P_{\mathcal{C}}^{\text {hor }}(g)$ is a D-telescopic $(C, C)$-quasi geodesic of $\left(\mathcal{C}, d_{t e l}^{D}\right)$.

Proof. By Lemma $4.4 P_{\mathcal{C}}^{\text {hor }}(g)$ is a $C_{4.4}(v)$-telescopic chain. Let us consider any two points $x, y$ in $G=P_{\mathcal{C}}^{h o r}(g)$. They are $r$-close to two points $x^{\prime}, y^{\prime}$ in $g$. We denote by $g_{x^{\prime} y^{\prime}}$ the subpath of $g$ between $x^{\prime}$ and $y^{\prime}$ and by $G_{x y}$ the subset of $G$ between $x$ and $y$. Since we now consider the $C_{4.4}(v)$-telescopic distance, $\left|G_{x y}\right|_{\text {vert }}^{C_{4.4}(v)}=\left|g_{x^{\prime} y^{\prime}}\right|_{\text {vert }}^{v}$. From Lemma 4.9 and since any two horizontal paths in $G$ are separated by a vertical segment of vertical length at least 1 , we then get $\left|G_{x y}\right|_{t e l}^{C_{4.4}(v)} \leq 2 C_{4.9}\left|g_{x^{\prime} y^{\prime}}\right|_{t e l}^{v}$. Since $g$ is a $v$-telescopic $(a, b)$ quasi geodesic, $\left|g_{x^{\prime} y^{\prime}}\right|_{t e l}^{v} \leq a d_{t e l}^{v}\left(x^{\prime}, y^{\prime}\right)+b$. But $d_{t e l}^{v}\left(x^{\prime}, y^{\prime}\right) \leq 2 r+d_{t e l}^{v}(x, y)$. Therefore:

$$
\left|G_{x y}\right|_{t e l}^{C_{4.4}(v)} \leq \underset{16}{2 C_{4.9}\left(a\left(2 r+d_{t e l}^{v}(x, y)\right)+b\right) .}
$$


Since all telescopic distances are quasi isometric (item (c) of Lemma 3.3), we so get the right inequality for the quasi geodesicity of $P_{\mathcal{C}}^{\text {hor }}(g)$. We leave it to the reader to work out the straightforward proof of the existence of constants $A \geq 1$ and $B \geq 0$ such that $1 / A d_{t e l}^{v}(x, y)-B \leq\left|G_{x y}\right|_{t e l}^{C_{4.4}(v)}$.

Proof of Theorem 4.6. Let $\widetilde{X}$ be a tree of hyperbolic spaces which satisfies the exponentialseparation property and such that each attaching-map from an edge-space into a vertexspace is a quasi isometry. Let $v \geq C_{3.6}$. Let $\mathcal{C}$ be any $v$-corridor. Since the attaching maps of the tree of hyperbolic spaces are all quasi isometries, $\operatorname{Core}(\mathcal{C})=\mathcal{T}$. It follows from Lemmas 3.7 and 4.4 that $\left(\mathcal{C}, d_{\text {tel }}^{C_{4.4}}\right)$ is a quasi geodesic metric space and that the $C_{4.4}$-vertical segments are exponentially separated. From item (b) of Lemma 3.8, this implies in particular that the endpoints of any diagonal with horizontal length greater than some constant $M$ are exponentially separated in all the directions of $\mathcal{T}$ outside a region with vertical width bounded by $2 C_{3.8}$.

Since $v \geq C_{3.6}$, there are $v$-vertical trees over $\mathcal{T}$ through any point of $\tilde{X}$. By Lemma 4.4, the horizontal projections of these trees to $\mathcal{C}$ give $C_{4.4}$-vertical trees over $\mathcal{T}$ through the points of $\mathcal{C}$.

Let $L \geq M$. Consider a length $L$ diagonal $h_{0}$ from the vertical boundary $B_{0}$ of $\mathcal{C}$ to some maximal $C_{4.4}$-vertical tree $T_{0}$ in $\mathcal{C}$, we assume for a while that such a $T_{0}$ exists. Then another length $L$ diagonal $h_{1}$ from $T_{0}$ to another maximal $C_{4.4}$-vertical tree $T_{1}$, and so on until arriving at a maximal $C_{4.4}$-vertical tree $T_{r}$ for which there exists no other $C_{4.4}$-vertical tree in $\mathcal{C}$ connected to $T_{r}$ by a length $L$ diagonal. Then, as a consequence of the exponential-separation property, there is an upper-bound $d_{0}(L, v)$ to the diagonal distance from $T_{r}$ to the other $v$-vertical boundary tree $B_{1}$ of $\mathcal{C}$. An ordered sequence composed of:

- the diagonals $h_{0}, h_{1}, \cdots, h_{r}$,

- a horizontal geodesic $h_{r+1}$ with $\left|h_{r+1}\right|_{h o r} \leq d_{0}(L, v)+1$ between $T_{r}$ and $B_{1}$ such that $d_{\text {vert }}\left(h_{r+1}, h_{r}\right) \leq d_{\text {vert }}\left(h, h_{r}\right)$ for any horizontal geodesic $h$ with the same properties,

- the $C_{4.4}$-vertical segments in $T_{0}, T_{1}, \cdots, T_{r}$ between the endpoints of the $h_{i}$ 's

gives to us the telescopic chain denoted by $\mathcal{P}$ in Theorem 4.6. Moreover, if $x$ and $y$ are two points in the vertical boundaries of $\mathcal{C}$, adding the $v$-vertical segments in $B_{0}$ and $B_{1}$ between the horizontal geodesics $h_{0}$ and $h_{r+1}$ and the points $x$ and $y$ gives the telescopic chain announced by item (c) as we are now going to check: we denote this last telescopic chain by $\mathcal{P}_{x}^{y}$.

Let $g$ be any $(a, b)$-quasi geodesic of $\widetilde{X}$ between $x$ and $y$. By Proposition 4.8, $g \subset$ $\mathcal{N}_{\text {tel }}^{C_{4.8}}(\mathcal{C})$. By item (c) of Lemma 3.3, there is some constant $Z(v)$ such that $g$ is $Z(v)$ Hausdorff close to some $v$-telescopic $\left(a^{\prime}, b^{\prime}\right)$-quasi geodesic chain: for the sake of simplification, we still denote by $g$ this $v$-telescopic chain and by $a$ and $b$ its constants of quasi geodesicity. From Lemma $4.10, \mathfrak{G} \equiv P_{\mathcal{C}}^{\text {hor }}(g)$ is a $D_{4.10}$-telescopic $\left(C_{4.10}, C_{4.10}\right)$-quasi geodesic of $\left(\mathcal{C}, d_{\text {tel }}^{D_{4.10}}\right)$.

The quasi geodesic $\mathfrak{G}$ intersects the vertical trees $T_{0}, T_{1}, \cdots$ of $\mathcal{C}$ : let $\mathfrak{G}_{0}$ be the shortest initial segment of $g$ that connects $x$ to $T_{0}$. From Proposition 4.7, $\mathfrak{G}_{0}$ is contained in the $C_{4.7}$-neighborhood of the union of the vertical segments $s_{0}, s_{1}$ from the endpoints of $\mathfrak{G}_{0}$ to those of $h_{0}$. From our observation above about the exponential separation of the endpoints of $h_{0}$, there is some $\kappa>0$ such that, outside the region in $\mathcal{C}$ centered at $h_{0}$ with vertical width $\kappa$, the horizontal geodesics between the vertical trees of the endpoints of $h_{0}$ have horizontal length greater than $3 C_{4.7}$. We so get a constant $K \equiv$ 
$K(v, L, a, b)>0$, not depending of the quasi geodesic nor on the corridor considered, such that $d_{t e l}^{H}\left(\mathfrak{G}_{0}, s_{0} \cup h_{0} \cup s_{1}\right) \leq K(v, L, a, b)$ (we recall that $d_{t e l}^{H}$ denotes the Hausdorff distance associated to the $D_{4.10}$-telescopic distance).

The same arguments apply for the subset $\mathfrak{G}_{i}$ between $T_{i-1}$ and $T_{i}$ until $i=r$. Since $\left|h_{r+1}\right|_{h o r} \leq d_{0}(L, v)+1$ and $h_{r+1}$ has been chosen to minimize the vertical distance between $h_{r}$ and all horizontal geodesics $h$ satisfying $|h|_{h o r} \leq d_{0}(L, v)+1$, we easily get a constant $K^{\prime} \equiv K^{\prime}(v, L, a, b)$ such that the concatenation of $h_{r+1}$ with

- the $v$-vertical segment in $B_{1}$ between $h_{r+1}$ and $y$ (the terminal point of $g$ ),

- the $C_{4.4}$-segment in $T_{r}$ between $h_{r}$ and $h_{r+1}$,

is at Hausdorff distance smaller than $K^{\prime}(v, L, a, b)$ from the subset of $\mathfrak{G}$ following the concatenation of the $\mathfrak{G}_{i}$ 's.

It follows that $\mathcal{P}_{x}^{y}$ is a $D_{4.10}$-telescopic chain between $x$ and $y$ with $d_{t e l}^{H}\left(g, \mathcal{P}_{x}^{y}\right) \leq$ $\max \left(K, K^{\prime}\right)$. We so proved items (b) and (c) of Theorem 4.6.

It remains to check item (a). It suffices to choose $a=1$ and $b=0$ and then apply what was proved just above: the chain $\mathcal{P}$ is at Hausdorff distance smaller than $\max \left(K(v, L, 1,0), K^{\prime}(v, L, 1,0)\right)$ from a geodesic. Moreover, by construction, the intersections of $\mathcal{P}$ with the strata are horizontal geodesics so that the non-properness of the strata cannot be used to shorten $\mathcal{P}$. From these observations, we easily get by classical arguments and computations that $\mathcal{P}$ is a $\left(d_{1}(L, v), d_{1}(L, v)\right)$-quasi geodesic as announced.

We now deal with the case where there is no $C_{4.4}$-vertical tree in $\mathcal{C}$ which is connected to $B_{0}$ by a length $L$ diagonal. Then, as was previously observed when dealing with the non-existence of a similar $C_{4.4}$-vertical tree between $T_{r}$ and $B_{1}$, there is an upper-bound, denoted here $d_{2}(L, v) \geq L$, on the diagonal distance between $B_{0}$ and $B_{1}$ : the maximum of the constants $d_{i}(L, v)+1, i=1,2,3$, gives the constant $D \geq L$ announced by Theorem 4.6. Let $g$ be any $(a, b)$-quasi geodesic of $\widetilde{X}$ between $x \in T$ and $y \in T^{\prime}$. By Proposition 4.8, $g \subset \mathcal{N}_{\text {tel }}^{C_{4.8}}(\mathcal{C})$. By item (c) of Lemma 3.3, there is some constant $Z(v)$ such that $g$ is $Z(v)$-Hausdorff close to some $v$-telescopic $\left(a^{\prime}, b^{\prime}\right)$-quasi geodesic chain: for the sake of simplification, we still denote by $g$ this $v$-telescopic chain and by $a$ and $b$ its constants of quasi geodesicity. From Lemma $4.10, \mathfrak{G} \equiv P_{\mathcal{C}}^{\text {hor }}(g)$ is a $D_{4.10}$-telescopic $\left(C_{4.10}, C_{4.10}\right)$-quasi geodesic of $\left(\mathcal{C}, d_{t e l}^{D_{4} .10}\right)$. The assertion of Theorem 4.6 in this case is then a straightforward consequence of Proposition 4.7 applied to $\mathfrak{G}$ if $\mathcal{C}$ contains a horizontal geodesic of length at least $D$. Otherwise $\mathfrak{G}$ is obviously in a bounded neighborhood of a telescopic chain $(h, s)$ with $|h|_{h o r} \leq D$ and the conclusion follows.

\section{Approximation OF QUASI GeOdesics: THE General CASE}

In order to give a simple statement, we added in Theorem 4.6 the restriction that the attaching-maps of the tree of spaces be quasi isometries, instead of requiring that they be quasi isometric embeddings. In this way, the elementary notion of a corridor (Definition 4.1) was sufficient to describe the quasi geodesics of the space. We now define generalized $v$-corridors, they substitute the corridors of Theorem 4.6 in order to obtain the more general statement we are looking for.

Definition 5.1. Let $(\tilde{X}, \mathcal{T}, \pi)$ be a tree of qi-embedded geodesic spaces. Let $v \geq C_{3.6}$.

If $h$ is a horizontal geodesic, a min-max generalized $v$-corridor $\mathcal{C}(h)$ through $h$ is a subset of $\widetilde{X}$ whose intersection with each stratum is either empty or a (possibly degenerate to a point) horizontal geodesic, satisfying the following properties

(a) $h$ is a maximal horizontal geodesic in $\mathcal{C}(h)$. 
(b) minimality condition: If $h_{1}$ is any horizontal geodesic in $\mathcal{C}(h)$ then there is a subgeodesic $h_{0}$ of $h$ such that any point in $h_{0}$ is connected to some point in $h_{1}$ by some $C_{4.4}(v)$-vertical segment in $\mathcal{C}(h)$, and conversely any point in $h_{1}$ is connected to some point in $h_{0}$ by some $C_{4.4}(v)$-vertical segment in $\mathcal{C}(h)$ : we say in this case that $h_{0}$ and $h_{1}$ are $C_{4.4}(v)$-paired in $\mathcal{C}(h)$.

(c) If $h_{0} \in X_{\alpha}$ and $h_{1} \in X_{\beta}$ are any two $C_{4.4}(v)$-paired horizontal geodesics in $\mathcal{C}(h)$ then for any $\gamma \in[\alpha, \beta], \mathcal{C}(h) \cap X_{\gamma}$ contains a horizontal geodesic $h_{2}$ which is $C_{4.4}(v)$-paired with both $h_{0}$ and $h_{1}$ in $\mathcal{C}(h)$.

(d) maximality condition: If $h_{0} \subset \mathcal{C}(h) \cap X_{\alpha}$ is a (possibly degenerate to a point) horizontal geodesic such that there exists $[\alpha, \beta] \in E(\mathcal{T})$ and a horizontal geodesic in $X_{\beta}$ which is $C_{4.4}(v)$-paired with both $h_{0}$ and some horizontal subgeodesic of $h$ in $\mathcal{C}(h)$ then there exists a horizontal geodesic $h_{1}$ in $\mathcal{C}(h) \cap X_{\beta}$ which is paired with $h_{0}$ and some horizontal subgeodesic of $h$ in $\mathcal{C}(h)$.

The union of all the endpoints of the maximal horizontal geodesics in $\mathcal{C}(h)$ decomposes as a union of disjoint maximal $C_{4.4}(v)$-vertical trees, called the vertical boundary of $\mathcal{C}(h)$.

Observe that the constant $C_{4.4}(v)$ appearing in the definition above loosens a little bit the definition of $v$-corridor since we allow the vertical boundaries to be $C_{4.4}(v)$-vertical trees instead of $v$-vertical trees. We require however that in the "interior" points in the generalized corridor are also connected by $C_{4.4}(v)$-vertical trees. Observe also that this definition is not empty in the sense that by Lemma 4.4, if we take $v$-vertical trees as vertical boundaries (which are in particular $C_{4.4}(v)$-vertical trees), the points in the generalized corridor will be connected by $C_{4.4}(v)$-vertical segments.

The notion of a min-max generalized $v$-corridor through a horizontal geodesic is sufficient if one only considers the problem of connecting two points $x, y$ in a same stratum: take any horizontal geodesic $h$ between $x$ and $y$ and then a neighborhood of a min-max generalized corridor through $h$ will contain the telescopic geodesics between $x$ and $y$. However, if we are given two points $x$ and $y$ such that no $C_{4.4}(v)$-vertical tree through $x$ intersects a stratum which is also intersected by a $C_{4.4}(v)$-vertical tree through $y$, then there is no min-max generalized corridor the vertical boundaries of which pass through both $x$ and $y$. However, in this case there exists a union of min-max generalized $v$-corridors which contains $x$ and $y$ in its vertical boundary, where a point is in the boundary of a union of min-max generalized $v$-corridors if it belongs to the vertical boundary of corridor in the union and to no other corridor..

Lemma 5.2. Let $(\widetilde{X}, \mathcal{T}, \pi)$ be a tree of qi-embedded geodesic spaces. Let $v \geq C_{3.6}$. For any two points $x$ and $y$ in $\widetilde{X}$, there exists a finite union of min-max generalized $v$-corridor which contains $x$ and $y$ in its vertical boundary.

Proof.

Theorem 5.3. Let $\tilde{X}$ be a tree of hyperbolic spaces which satisfies the exponentialseparation property. Then for any $v \geq C_{3.6}$, for any $L$ greater than some critical constant, for any $a \geq 1$ and $b \geq 0$ there are $E \equiv E(v) \geq v, D \equiv D(L, v) \geq L$ and $C \equiv C(L, v, a, b) \geq 0$ such that the following holds:

For any two distinct $v$-vertical trees $T_{0}$ and $T_{1}$ in $\tilde{X}$ which are at diagonal distance at least $D$, for any generalized $v$-corridor $\mathcal{C}$ whose vertical boundaries contain $T_{0}$ and $T_{1}$, there is an E-telescopic chain $\mathcal{P}$ between $T_{0}$ and $T_{1}$ in $\mathcal{C}$ satisfying the following properties:

(a) $\mathcal{P}$ is a $(D, D)$-quasi geodesic which connects $T_{0}$ and $T_{1}$.

(b) If we denote by $\mathcal{C}_{i}$ the min-max generalized $v$-corridors which form $\mathcal{C}$ (see Definitions 5.1) then at the exception of at most one in each $\mathcal{C}_{i}$, each horizontal geodesic 
in $\mathcal{P}$ is a length $L$ diagonal. All the other horizontal geodesics in $\mathcal{P}$ have horizontal length smaller than $D$.

(c) For any $(a, b)$-quasi geodesic $g$ in $\widetilde{X}$ with endpoints in $T_{0}$ and $T_{1}$, if $t_{i}$ denotes the $v$-vertical segment in $T_{i}(i=0,1)$ from the endpoint of $\mathcal{P}$ to the endpoint of $g$ then $\left(*, t_{0}^{-1}, \mathcal{P}, t_{1}, *\right)$, where $*$ denotes the trivial horizontal path, is a $(D, D)$-quasi geodesic E-telescopic chain whose Hausdorff distance from $g$ in $\left(\widetilde{X}, d_{\tilde{X}}\right)$ is bounded above by $C$.

If $T_{0}$ and $T_{1}$ are at diagonal distance smaller than $D$ then any $(a, b)$-quasi geodesic $g$ in $\widetilde{X}$ with endpoints in $T_{0}$ and $T_{1}$ is contained in the telescopic $C$-neighborhood of $T_{0} \cup T_{1}$. More precisely, $g$ is at Hausdorff distance smaller than $C$ from a telescopic chain of the form $\left(*, t_{0}^{-1}, h_{0}, t_{1}, *\right)$ where $h_{0}$ is a (possibly degenerate to a point) horizontal geodesic in $\mathcal{C}$ with horizontal length smaller than $D$, and $t_{i}$ is the $v$-vertical segment in $T_{i}$ from $h_{0}$ to $g$.

Proof of Theorem 5.3. We first need an adaptation to this more general setting of some of the lemmas and propositions given for proving Theorem 4.6:

Proposition 5.4. Lemma 4.4, Proposition 4.7 and Proposition 4.8 remain true for generalized $v$-corridors with $v \geq C_{3.6}$.

There is nothing to prove with respect to Lemma 4.4 and Proposition 4.7. We refer the reader to Section 9.6 for the proof of the adaptation of Proposition 4.8 to generalized corridors.

Lemma 5.5. Let $\tilde{X}$ be a tree of hyperbolic spaces.

For any $v \geq C_{3.6}$, for any $a \geq 1$ and $b \geq 0$ there exists $C \geq 0$ such that if $g$ is any $(a, b)$-quasi geodesic, if $\mathcal{C}$ is any generalized $v$-corridor the vertical boundaries of which pass through the endpoints of $g$, then there is $a\left(a, b+D_{3.6}\right)$-quasi geodesic $G$ with $d_{\widetilde{X}}^{H}(g, G) \leq C$ and $\pi(G) \subset \pi(\mathcal{C})$.

Proof. Let $\gamma \in \mathcal{T}$ be an endpoint of $\pi(\mathcal{C})$. Assume that $g^{\prime}$ is a maximal subset of $g$ with endpoints in $X_{\gamma}$ and such that $\pi\left(g^{\prime}\right) \cap \pi(\mathcal{C})=\gamma$. Then, since $v \geq C_{3.6}$, Lemma 3.6 tells us that the endpoints of $g^{\prime}$ are $D_{3.6}$-close with respect to the horizontal distance. Since $g$ is a $(a, b)$-quasi geodesic, $g^{\prime}$ is $\left(a D_{3.6}+b\right)$-close to $X_{\gamma}$ with respect to the telescopic distance. Substituting $g^{\prime}$ by a horizontal geodesic between its endpoints and repeating this substitution for all the subsets of $g$ like $g^{\prime}$ yields a quasi geodesic as announced.

With the above adaptations in mind, the proof of Theorem 5.3 is now almost a duplicate of the proof of Theorem 4.6: in a first step, in each min-max generalized corridor which forms $\mathcal{C}$ build a telescopic path $\mathcal{P}_{i}$ in the same way than was built the telescopic path $\mathcal{P}$ of Theorem 4.6, which minimizes the telescopic distance from one vertical boundary tree to another one. The concatenation of these $\mathcal{P}_{i}$ with vertical segments between the endpoints of any two consecutive ones yields the announced telescopic path $\mathcal{P}$ : as announced, it only depends on the vertical trees in the boundary of $\mathcal{C}$ that it connects.

\section{WEAK RELATIVE HYPERBOLICITY}

The aim of this section is to prove Theorem 2.16. An intermediate result is Theorem 6.1 which generalizes Bestvina-Feighn's combination to non-proper hyperbolic spaces. Bowditch proposed such a generalization in [4].

Theorem 6.1. Let $\tilde{X}$ be a tree of hyperbolic spaces which satisfies the exponentialseparation property. Then $\tilde{X}$ is a Gromov-hyperbolic metric space. 
Proof. We begin by proving the

Theorem 6.2. Let $\tilde{X}$ be a tree of hyperbolic spaces which satisfies the exponentialseparation property. For any $a \geq 1$ and $b \geq 0$ there exists $C \geq 0$ such that $(a, b)$-quasi geodesic bigons are $C$-thin.

Proof of Theorem 6.2. By item (c) of Lemma 3.3, it suffices to prove Theorem 6.2 for $C_{3.6}$-telescopic $(a, b)$-quasi geodesic bigons. Let $g_{0}, g_{1}$ be the two sides of a $C_{3.6}$-telescopic $(a, b)$-quasi geodesic bigon. By Lemma 5.2 (the points $x$ and $y$ in the statement of this lemma are the endpoints of the bigon $g_{0} \cup g_{1}$ ) and Theorem 5.3 (Remark 4.2 and Theorem 4.6 suffice in the case where the attaching-maps of $\widetilde{X}$ are quasi isometries), there is $E_{5.3} \geq C_{3.6}$ and an $E_{5.3}$-telescopic chain $\mathcal{P}$ such that for $i=0,1$ we have $d^{H}\left(g_{i}, \mathcal{P}\right) \leq C_{5.3}$ in the case where the diagonal distance between two $v$-vertical trees passing through $x$ and $y$ is at least $L$. Hence $d^{H}\left(g_{0}, g_{1}\right) \leq 2 C_{5.3}$ in this case and Theorem 6.2 is proved. In the case where the diagonal distance is smaller than $L$, since the $g_{i}$ 's have the same endpoints, the last assertion of Theorem 5.3 yield the same conclusion.

The following lemma was first indicated to the author by I. Kapovich:

Lemma 6.3. [10] Let $(X, d)$ be a $(r, s)$-quasi geodesic space. If for any $r^{\prime} \geq r, s^{\prime} \geq s$, there exists $\delta\left(r^{\prime}, s^{\prime}\right)$, such that $\left(r^{\prime}, s^{\prime}\right)$-quasi geodesic bigons are $\delta\left(r^{\prime}, s^{\prime}\right)$-thin, then $(X, d)$ is a $2 \delta(r, 3 s)$-hyperbolic space.

Theorem 6.2 together with Lemma 6.3 imply Theorem 6.1.

Proof of Theorem 2.16: Let $\left(\mathcal{T},\left\{\left(X_{e}, \mathcal{P}_{e}\right)\right\},\left\{\left(X_{v}, \mathcal{P}_{v}\right)\right\},\left\{\jmath_{e}\right\}\right)$ be a tree of weakly relatively hyperbolic spaces. Since $\left(\mathcal{T},\left\{\widehat{X}_{e}\right\},\left\{\widehat{X}_{v}\right\},\left\{\widehat{\jmath}_{e}\right\}\right)$ satisfies the exponential-separation property, by Theorem $6.1,\left(\mathcal{T},\left\{\widehat{X}_{e}\right\},\left\{\widehat{X}_{v}\right\},\left\{\widehat{\jmath}_{e}\right\}\right)$ is hyperbolic. This is exactly equivalent to (the geometric realization of) $\left(\mathcal{T},\left\{\left(X_{e}, \mathcal{P}_{e}\right)\right\},\left\{\left(X_{v}, \mathcal{P}_{v}\right)\right\},\left\{\jmath_{e}\right\}\right)$ being weakly hyperbolic relatively to the family composed of all the parabolic subspaces of the edge- and vertex-spaces and Remark 2.17 is proved. Since the attaching-maps of the trees of spaces are assumed to be pair-maps, the parabolic subspaces of the edge-spaces are mapped into the parabolic subspaces of the vertex-spaces. Thus the parabolic subspaces of the edge-spaces can be removed from the previous family and (the geometric realization of) $\left(\mathcal{T},\left\{\left(X_{e}, \mathcal{P}_{e}\right)\right\},\left\{\left(X_{v}, \mathcal{P}_{v}\right)\right\},\left\{\jmath_{e}\right\}\right)$ is weakly hyperbolic relatively to the family composed of all the parabolic subspaces of the vertex-spaces.

\section{Strong RELATIVE HyPeRBolicity}

The goal in this section is to prove Theorem 2.21. We need some preliminary lemmas and a proposition the proof of which is postponed to Section 9.7.

Consider any induced tree of parabolic spaces. Since in $\widehat{X}$ a cone has been put over each parabolic space, this induced tree of parabolic spaces is naturally assimilated to a tree whose vertices are the vertices of the cones over the parabolic spaces. By definition of $\widehat{\jmath}_{e}$, this tree is further assimilated to a 0-vertical tree: in order not to add unnecessary additional vocabulary, we call "induced tree of parabolic spaces" this 0-vertical tree of cone-vertices.

The following lemma is a straightforward consequence of the strong exponential-separation property:

Lemma 7.1. There exists $C \geq 0$ such that any two induced trees of parabolic spaces which intersect a same stratum are connected by a diagonal (see Definition 4.5) of horizontal 
length greater or equal to 1 , the endpoints of which are exponentially separated in all the directions outside a region whose vertical width is smaller than $C$.

Lemma 7.2. For any $a \geq 1$ and $b \geq 0$ there exists $C \geq 0$ such that if $g, g^{\prime}$ are two $(a, b)$ quasi geodesics of $\widehat{X}$ between two induced trees of parabolic spaces $L_{1}, L_{2}$ then $g, g^{\prime}$ admit decompositions $g=g_{1} g_{2} g_{3}$ and $g^{\prime}=g_{1}^{\prime} g_{2}^{\prime} g_{3}^{\prime}$ with the following properties: $g_{1} \subset \mathcal{N}_{\widehat{X}}^{C}\left(L_{1}\right)$, $g_{1}^{\prime} \subset \mathcal{N}_{\widehat{X}}^{C}\left(L_{1}\right), g_{3} \subset \mathcal{N}_{\widehat{X}}^{C}\left(L_{2}\right), g_{3}^{\prime} \subset \mathcal{N}_{\widehat{X}}^{C}\left(L_{2}\right)$ and $d_{\widehat{X}}^{H}\left(g_{2}, g_{2}^{\prime}\right) \leq C$. If $g$ and $g^{\prime}$ have the same endpoints then $d_{\widehat{X}}^{H}\left(g, g^{\prime}\right) \leq C$.

Proof. This is an easy consequence of Theorem 5.3. For simplicity assume that the attaching-maps of $\widehat{X}$ are quasi isometries so that Theorem 4.6 can be applied. The induced trees of parabolic spaces bound a corridor. Both $g$ and $g^{\prime}$ are approximated by two chains $G$ and $G^{\prime}$ which only possibly differ by their first and last vertical segments in $L_{1}$ and $L_{2}$. These last vertical segments are where $g$ and $g^{\prime}$ are not necessarily close one to each other if they don't have the same endpoints but are close to the given vertical trees. As written before, the extension to the general case where there is not a corridor, but only a generalized corridor, between the two induced trees, is easily dealt with by using Theorem 5.3 instead of Theorem 4.6.

We denote by $C(\widehat{X})$ the metric space obtained from the geometric realization of $\left(\mathcal{T},\left\{\widehat{X}_{e}\right\},\left\{\widehat{X}_{v}\right\},\left\{\widehat{J}_{e}\right\}\right)$ by putting a cone over (the geometric realization of) each induced tree of parabolic spaces, that is over each one of the associated tree of cone-vertices (see above). We recall that the acronym BPP below stands for Bounded-Parabolic Penetration property, see Definition 2.3.

Lemma 7.3. For any $v \geq C_{3.6}$, for any $a \geq 1$ and $b, r \geq 0$ there exists $C \geq 0$ such that if $g_{1}, g_{2}$ are two $(a, b)$-quasi geodesics of $C(\widehat{X})$, the terminal points of which are at most 1-apart in $\widehat{X}$, and with same initial point in $\widehat{X}$, if $\mathcal{C}$ is a generalized v-corridor whose vertical boundaries pass through the endpoints of $g_{1}$, if traces $\widehat{g}_{i}$ 's of the $g_{i}$ 's in $\widehat{X}$ satisfy $\widehat{g}_{i} \subset \mathcal{N}_{\widehat{X}}^{r}(\mathcal{C})$ for $i=1,2$ then $d_{C(\widehat{X})}^{H}\left(g_{1}, g_{2}\right) \leq C$. Furthermore, if $g_{1}$ and $g_{2}$ do not backtrack then they satisfy the two conditions required by the BPP with a constant D depending on $v, a, b, r$.

We emphasize that this proposition is false if one only requires a bound on the distance in $C(\widehat{X})$ from the $g_{i}$ 's to $\mathcal{C}$.

Proof. For simplicity we assume that $\mathcal{C}$ is a corridor, the adaptation to generalized corridors is straightforward. Moreover, by item (c) of Lemma 3.3, the passages of $g_{1}$ and $g_{2}$ in $\widehat{X}$ can be approximated by telescopic quasi geodesics: for the sake of simplification, we act as these passages were telescopic quasi geodesics. We consider the horizontal quasi projections on $\mathcal{C}$ of the maximal subsets of $g_{1}, g_{2}$ which belong to $\widehat{X}$. From Lemma 4.10, these projections are $\left(C_{4.10}, C_{4.10}\right)$-quasi geodesics. From Lemma 7.1 on the one hand and Lemma 3.7 on the other hand, there is $K$, depending on $r$ and $C_{4.4}(0)$ (recall indeed that the induced trees of parabolic spaces are assimilated to 0-vertical trees), such that the horizontal quasi projections of the induced trees of parabolic spaces (that is the associated trees of cone-vertices) are $K$-vertical trees, for which there exists a constant $L$ playing the rôle of the constant $t_{2.20}$. It is equivalent to prove the announced properties for the bigon $g_{1}, g_{2}$ with respect to the trees of cone-vertices than to prove them for the above projections on $\mathcal{C}$.

If $g_{1}, g_{2}$ go through the same trees of cone-vertices, then their horizontal quasi projections on $\mathcal{C}$ satisfy the same property with respect to the horizontal quasi projections 
of the trees of cone-vertices. From Lemma 7.2, the "bigon" obtained by projection to the generalized corridor is thin. Moreover the points where the projections of $g_{1}$ and $g_{2}$ penetrate a given tree of cone-vertices are close, because either they are close to the diagonal preceding this tree, or they leave a same tree of cone-vertices: in this last case we are done by the existence of the constant $L$ above (the analog on the corridor of the constant $\left.t_{2.20}\right)$. Let us now assume that $g_{1}$ enters in a tree of cone-vertices $S$ but $g_{2}$ does not. Of course this also holds for the respective projections on $\mathcal{C}$. We then distinguish three cases:

First case: the exit point of $g_{1}$ is followed by a diagonal with horizontal length greater than some constant (depending on the constants of hyperbolicity and exponential separation). Then (the projection of) $g_{2}$ has to go to a bounded neighborhood of this diagonal, this is Theorem 4.6. It remains before in a bounded horizontal neighborhood of the tree of cone-vertices, the bound depending on $a, b$ and $r$ (since the constants of quasigeodesicity of the projections depend on $r$ ). Thus the vertical length of the passage of $g_{1}$ through this tree is bounded above by a constant depending on $a, b$ and $r$.

Second case: the exit point of $g_{1}$ is followed by another tree of cone-vertices. Thanks to the existence of the constant $L$ and Lemma 7.1, we can follow the same arguments as above, appealing to Proposition 4.7 rather than directly Theorem 4.6. We leave the reader work out details and computations.

Third case: the exit point of $g_{1}$ is followed by a horizontal geodesic with horizontal length bounded above by the constant of the first case. In this case, this horizontal geodesic ends at the vertical boundary of $\mathcal{C}$. The entrance-point of $g_{1}$ in $S$ is close to a point in $g_{2}$. Since $g_{2}$ is a $(a, b)$-quasi geodesic and $g_{2}$ does not pass through $S$, it cannot happen that the passage of $g_{1}$ though $S$ is a long passage at small horizontal distance from the considered vertical boundary. Thus, if it is a long passage then there is a stratum which is closest to the entrance-point of $g_{1}$ in $S$ and where the horizontal distance between $S$ and the considered vertical boundary is smaller than the critical constant. From Proposition 4.7, $g_{2}$ lies in a bounded neighborhood of $S$ until reaching this stratum. Once again, this gives an upper-bound on the vertical length of $S$.

The proof of Lemma 7.3 now follows in an easy way: to conclude for the BPP, we need of course the fact that the horizontal metrics on the strata satisfy the BPP.

Proposition 7.4. With the assumptions of Lemma 7.3: For any $v \geq C_{3.6}$, for any $a \geq 1$ and $b \geq 0$ there exist $C \geq 1$ and $D>0$ such that, if $x_{0}, x_{1}, \cdots, x_{n}$ are consecutive points in some tree of cone-vertices $L$, which lie outside the horizontal D-neighborhood of a generalized $v$-corridor $\mathcal{C}$, and if the vertical distance between the strata of $x_{0}$ and $x_{n}$ is greater than $C$, then no non-backtracking $(a, b)$-quasi geodesic of $C(\widehat{X})$ with both endpoints in the horizontal D-neighborhood of $\mathcal{C}$ contains the cone over $\left\{x_{0}, x_{n}\right\}$.

See proof in subsection 9.7.

Proof of Theorem 2.21. Let $g, g^{\prime}$ be two non-backtracking $(a, b)$-quasi geodesics of $C(\widehat{X})$ with same initial point, and with terminal points at most 1-apart in $\widehat{X}$. We assume for simplicity that the attaching-maps of $\widehat{X}$ are quasi isometries, the adaptation to the general case is easy. There is a corridor $\mathcal{C}$ (in the whole generality only a generalized corridor) the vertical boundaries of which pass through the initial and terminal points of $g$.

Let $p$ be a passage of $g$ (resp. of $g^{\prime}$ ) through the cone over a subset $S$ of an induced tree of parabolic spaces (that is a tree of cone-vertices) outside the $D_{7.4}$-neighborhood of $\mathcal{C}$ in 
$\widehat{X}$. From Proposition 7.4, substituting $p$ by $S$ yields a non-backtracking $\left(\kappa(a, b), \kappa^{\prime}(a, b)\right)$ quasi geodesics $h$ (resp. $h^{\prime}$ ) of $C(\widehat{X})$, with $\kappa(a, b)=C_{7.4} * a$ and $\kappa^{\prime}(a, b)=C_{7.4} *(b+1)$, such that $d_{C(\widehat{X})}^{H}(g, h) \leq 1$ (resp. $\left.d_{C(\widehat{X})}^{H}\left(g^{\prime}, h^{\prime}\right) \leq 1\right)$. We can thus assume that all passages like $p$ have been suppressed in $h$ and $h^{\prime}$ as above.

By Proposition 4.8, the subsets of $h$ and $h^{\prime}$ between two trees of cone-vertices are contained in the horizontal $C_{4.8}$-neighborhood of a corridor between these trees. Thus $h$ and $h^{\prime}$ are contained in the $D_{7.4}+C_{4.8}$-neighborhood of $\mathcal{C}$ in $\widehat{X}$. From Lemma $7.3, h, h^{\prime}$ satisfy the BPP. The conclusion for $g, g^{\prime}$ follows.

The proof of the hyperbolicity follows the same scheme. If $g, g^{\prime}$ form a $(a, b)$-quasi geodesic bigon of $C(\widehat{X})$, one first substitutes it by a non-backtracking $(a, b)$-quasi geodesic bigon $g_{0}, g_{0}^{\prime}$ with $d_{C(\widehat{X})}^{H}\left(g, g_{0}\right) \leq b, d_{C(\widehat{X})}^{H}\left(g^{\prime}, g_{0}^{\prime}\right) \leq b$. The line of the arguments thereafter is the same than above: at the end, Lemma 7.3 gives the thinness of the quasi geodesic bigons instead of the BPP. As in Section 6, the hyperbolicity follows from Lemma 6.3.

Remark 7.5. The hyperbolicity of the coned space $C(\widehat{X})$ follows from the quasi convexity of the trees of cone-vertices and from the arguments developed for proving Proposition 1 of [22]. However we re-proved it above when listing the arguments for checking the BPP.

\section{Proof of Proposition 4.7}

Conventions: The constants of hyperbolicity and of quasi isometry are chosen sufficiently large to satisfy the conclusions of Lemma 3.8, and also sufficiently large so that computations make sense. Moreover the horizontal subsets of the $(a, b)$-quasi geodesics considered will be assumed to be horizontal geodesics. The hyperbolicity of the strata gives, for any $a \geq 1$ and $b \geq 0$, a positive constant $C(a, b)$ such that any $(a, b)$-quasi geodesic $g$ may be substituted by another one $g^{\prime}$ with $d_{\widetilde{X}}^{H}\left(g, g^{\prime}\right) \leq C(a, b)$ and satisfying this latter property.

In the proofs of the various intermediate statements, when referring to a constant provided by an earlier result we will sometimes indicate between parentheses the values of some of the parameters from which it depends.

Our first lemma is about quasi geodesics. It holds not only in a corridor but in the whole tree of hyperbolic spaces.

Lemma 8.1. Let $(\tilde{X}, \mathcal{T}, \pi)$ be a tree of hyperbolic spaces which satisfies the exponentialseparation property. For any $a \geq 1, b \geq 0$ and for any $v \geq C_{3.6}$ there exist $C \geq 0$ and $D \geq 0$ such that, if $g$ is a $(a, b)$-quasi geodesic in $\widetilde{X}$, if $[x, y] \subset g \cap X_{\alpha}$ satisfies $d_{\text {hor }}(x, y) \geq C$ then for any $\mathcal{T}$-geodesic $\omega$ starting at $\alpha$ with $|\omega|_{\mathcal{T}} \geq D+n t_{0}, n \geq 1$, we have $d_{\text {hor }}(\omega x, \omega y) \geq \lambda^{n} d_{\text {hor }}(x, y)$.

Proof. We denote by $\lambda>1, M, t_{0} \geq 1$ the constants of hyperbolicity and by $\lambda_{+}, \mu$ the constants of quasi isometry. Let us choose $n_{\star}(a)$ such that $\frac{a}{\lambda^{n_{\star}}}<1$. Solving the inequality $e>a\left(\frac{1}{\lambda^{n_{\star}}} e+2 n_{\star} t_{0}\right)+b$ gives us $e(a, b) \geq \frac{2 a n_{\star} t_{0}+b}{1-a \frac{1}{\lambda_{\star}}}$.

Claim: If $d_{\text {hor }}(x, y) \geq e(a, b)$, if $x^{\prime}, y^{\prime}$ are the endpoints of two $v$-vertical segments $s, s^{\prime}$ of vertical length $n_{\star} t_{0}$, starting at $x$ and $y$ and with $\pi(s)=\pi\left(s^{\prime}\right)$, then for any $\mathcal{T}$-geodesic $\omega_{0}$ such that $\omega_{0} \pi(s)$ is a $\mathcal{T}$-geodesic and $\left|\omega_{0}\right|_{\mathcal{T}}=t_{0}, d_{\text {hor }}\left(\omega_{0} x^{\prime}, \omega_{0} y^{\prime}\right) \geq \lambda d_{\text {hor }}\left(x^{\prime}, y^{\prime}\right)$ holds. Proof of Claim: Assume the existence of $\omega$ with $|\omega|_{\mathcal{T}}=n_{\star} t_{0}$ such that for some $x^{\prime}, y^{\prime}$ with $x \in \omega x^{\prime}, y \in \omega y^{\prime}$ and $d_{\text {hor }}\left(x^{\prime}, y^{\prime}\right) \geq M, d_{\text {hor }}(x, y) \geq \lambda^{n_{\star}} d_{\text {hor }}\left(x^{\prime}, y^{\prime}\right)$ holds. Then $\frac{1}{\lambda_{\star}} e+2 n_{\star} t_{0}$ is the telescopic length of a telescopic chain between $x$ and $y$. But the inequality given at the beginning of the proof tells us that the existence of such a telescopic chain is a contradiction with the fact that $g$ is a $v$-telescopic $(a, b)$-quasi geodesic. Therefore, if 
$d_{h o r}(x, y) \geq e(a, b)$ and $d_{h o r}(x, y) \geq \lambda_{+}^{n_{\star}}(M+\mu)$ (this last inequality is to assert that $d_{\text {hor }}\left(x^{\prime}, y^{\prime}\right) \geq M$ - see above), then $d_{\text {hor }}\left(x^{\prime}, y^{\prime}\right)$ does not increase after $t_{0}$ in the direction of the $v$-vertical segments $s, s^{\prime}$. The claim follows from the exponential separation of the $v$-vertical segments.

From the inequality given by the Claim, since $d_{h o r}\left(x^{\prime}, y^{\prime}\right) \geq \lambda_{+}^{-n_{\star}}\left(d_{h o r}(x, y)+\mu\right)$, we easily compute an integer $N_{\star}$ such that, if $\omega_{0}$ is as in the Claim but with length $N_{\star} t_{0}$ then $d_{\text {hor }}\left(\left[\omega_{0} \pi(s)\right] x,\left[\omega_{0} \pi(s)\right] y\right) \geq \lambda d_{\text {hor }}(x, y)$. Setting $D=N_{\star} t_{0}$ and $C(a, b)=e(a, b)$, the constant computed above, we get the lemma.

Notations: $\delta$ a fixed non negative constant, $(\tilde{X}, \mathcal{T}, \pi)$ a tree of $\delta$-hyperbolic spaces, $w \geq C_{3.6}$ and $v \geq C_{4.4}(w)$ two constants, $\lambda>1, M, t_{0} \geq 1$ the associated constants of hyperbolicity, $\lambda_{+}, \mu$ the associated constants of quasi isometry.

Lemma 8.2. For any $a \geq 1, b \geq 0$, there exists $C \geq 0$ such that if $\mathcal{C}$ is a generalized $w$-corridor with exponentially separated v-vertical segments, if $g$ is a v-telescopic chain which is a $(a, b)$-quasi geodesic of $\left(\mathcal{C}, d_{\text {tel }}^{v}\right)$, if the endpoints $x, y$ of $g$ both lie in a same stratum $X_{\alpha}$, if $d_{\text {hor }}(x, y) \geq C$ then, for any $\mathcal{T}$-geodesic $\omega$ starting at $\alpha$ with $|\omega|_{\mathcal{T}} \geq C+n t_{0}$, $n \geq 1$, and $\omega \cap \pi(g)=\{\alpha\}$, we have:

$$
d_{h o r}(\omega x, \omega y) \geq \lambda^{n} d_{h o r}(x, y) .
$$

Proof. Let us observe that, if $[p, q]$ is any horizontal geodesic in $g$ then the $v$-vertical trees of $p$ and $q$ bound a horizontal geodesic $\left[p^{\prime}, q^{\prime}\right]$ in $[x, y]$.

Claim: If $d_{\text {hor }}\left(p^{\prime}, q^{\prime}\right) \geq C$ te with $C t e \equiv \lambda_{+}^{t_{0}}\left(C_{8.1}+t_{0}+\mu\right)$ then for any $\omega$ as given by the current Lemma with $|\omega|_{\mathcal{T}} \geq D_{8.1}+t_{0}, d_{\text {hor }}\left(\omega p^{\prime}, \omega q^{\prime}\right) \geq \lambda d_{\text {hor }}\left(p^{\prime}, q^{\prime}\right)$.

Proof of Claim: If $p^{\prime}$ and $q^{\prime}$ are not exponentially separated in the direction of $p, q$ after $t_{0}$, then, because of the exponential-separation property, they are exponentially separated after $t_{0}$ in the direction of $\omega$, which yields the announced inequality. Let us assume that $p^{\prime}, q^{\prime}$ are separated after $t_{0}$ in the direction of $\left[\pi\left(p^{\prime}\right), \pi(p)\right]$. Thus $d_{\text {hor }}\left(r p^{\prime}, r q^{\prime}\right) \geq$ $\lambda^{n} d_{\text {hor }}\left(p^{\prime}, q^{\prime}\right)$ for a $\mathcal{T}$-geodesic $r$ with $|r|_{\mathcal{T}}=n t_{0}$ and $r \cap \omega=\{\alpha\}$. Therefore $d_{\text {hor }}(p, q) \geq$ $C_{8.1}+t_{0}$. Lemma 8.1 then implies that $p, q$ are exponentially separated in the direction of $\left[\pi(p), \pi\left(p^{\prime}\right)\right]$ after $D_{8.1}+t_{0}$, and the claim is proved.

There is a finite decomposition of $[x, y] \subset X_{\alpha}$ in subgeodesics $\left[p_{j}^{\prime}, q_{j}^{\prime}\right]$ with disjoint interiors such that each $\left[p_{j}^{\prime}, q_{j}^{\prime}\right]$ connects two $v$-vertical trees through the endpoints of a horizontal geodesic in $g$. We denote by $I_{D}$ the set of $\left[p_{j}^{\prime}, q_{j}^{\prime}\right]^{\prime}$ 's with $d_{h o r}\left(p_{j}^{\prime}, q_{j}^{\prime}\right) \geq C t e$ and by $I_{C}$ the set of the others. Let us choose an integer $n \geq 1$. We consider a stratum $X_{\beta}$ with $d_{\mathcal{T}}(\beta, \alpha)=D_{8.1}+n t_{0}$. Let $h$ be the horizontal geodesic in $\mathcal{C} \cap X_{\beta}$ which connects the two $v$-vertical trees through $x$ and $y$. Assume that the endpoints of $h$ are exponentially separated after $t_{0}$ in the direction of $[\beta, \alpha]$. Then:

$$
\lambda^{n}\left|I_{D}\right|_{\text {hor }} \leq|h|_{\text {hor }} \leq \lambda^{-n}\left(\left|I_{D}\right|_{\text {hor }}+\left|I_{C}\right|_{\text {hor }}\right)
$$

so that

$$
\left|I_{C}\right|_{h o r} \geq \frac{\lambda^{n}-\lambda^{-n}}{\lambda^{-n}}\left|I_{D}\right|_{h o r}
$$

and consequently, since $d_{\text {hor }}(x, y)=\left|I_{D}\right|_{\text {hor }}+\left|I_{C}\right|_{\text {hor }}$,

$$
\left|I_{C}\right|_{\text {hor }} \geq \frac{X(n)}{1+\underset{25}{X(n)}} d_{\text {hor }}(x, y)
$$


with $X(n)=\frac{\lambda^{n}-\lambda^{-n}}{\lambda^{-n}}$. Since $\lim _{n \rightarrow+\infty} \frac{X(n)}{1+X(n)}=1$, there is $n_{\star} \geq 0$ such that for any $n \geq n_{\star}$,

$$
\left|I_{C}\right|_{h o r} \geq \frac{1}{2} d_{\text {hor }}(x, y) \text {. }
$$

But, by definition, the horizontal length of each subgeodesic in $I_{C}$ is smaller than Cte. Thus the number of elements in $I_{C}$ is at least the integer part of $\frac{1}{2 C t e} d_{\text {hor }}(x, y)+1$. Furthermore, since $g$ is a $v$-telescopic chain, the telescopic length of any subset of $g$ containing $j$ horizontal geodesics is at least $(j-1)$. We so obtain:

$$
|g|_{\text {tel }}^{v} \geq \frac{1}{2 C t e} d_{h o r}(x, y) \text {. }
$$

On the other hand:

$$
d_{t e l}^{v}(x, y) \leq \lambda^{-n} d_{h o r}(x, y)+2 n t_{0}
$$

since there is a $v$-telescopic chain between $x$ and $y$ the telescopic length of which is given by the right-hand side of the above inequality. Since $g$ is a $(a, b)$-quasi geodesic, the last two inequalities give $n_{\star \star} \geq 0$ such that for $n \geq n_{\star \star}$ :

$$
d_{\text {hor }}(x, y) \leq \frac{2 a n t_{0}+b}{\frac{1}{2 C t e}-a \lambda^{-n}} .
$$

Taking the maximum of $n_{\star}, n_{\star \star}$ and the above upper-bound for $d_{h o r}(x, y)$, we get the announced constant in the case where the endpoints of the horizontal geodesic $h$ above are exponentially separated in the direction of $[\beta, \alpha]$. If not, there are in all the other directions so that we easily get a constant $N \geq 0$ such that $d_{h o r}(\omega x, \omega y) \geq \lambda d_{h o r}(x, y)$ for any $\mathcal{T}$-geodesic $\omega$ with $|\omega|_{\mathcal{T}}=N t_{0}$ and $[\pi(x), \pi(h)] \subset \omega$. Lemma 8.2 is then easily deduced.

As a consequence we have:

Corollary 8.3. For any $a \geq 1, b \geq 0$ and $d \geq M$, there exists $C \geq d$ such that if $\mathcal{C}$ is a generalized $w$-corridor with exponentially separated $v$-vertical segments, if $g$ is any $v$-telescopic chain which is a $(a, b)$-quasi geodesic of $\left(\mathcal{C}, d_{\text {tel }}^{v}\right)$, if $x, y$ are the endpoints of two $v$-vertical segments $s, s^{\prime}$ over a same edge-path in $\mathcal{T}$, with $\pi(s) \cap \pi(g)=\{\alpha\}$ and such that $d_{\text {hor }}\left(s, s^{\prime}\right) \leq d$, then $d_{\text {hor }}(x, y) \leq C$.

Remark 8.4. At this point, we would like to notice that Lemma 8.2 is similar to Lemma 6.7 of [10]. However in addition of some misprints, a slight mistake took place there in the proof of the Lemma. Indeed the inequality (1) in the proof of Lemma 8.2 is true here, in the generalized corridor, but there the constant $\lambda$ should have been modified to take into account the so-called "cancellations".

Lemma 8.5. For any $r \geq 0$, there exists $C \geq 0$ such that if $\mathcal{C}$ is a generalized $w$ corridor with exponentially separated $v$-vertical segments, if $x$ and $y$ are the endpoints of a r-vertical segment $s$ in $\mathcal{C}$, if the intersection-point $z$ of some $v$-vertical tree through $y$ in $\mathcal{C}$ with the stratum $X_{\pi(x)}$ satisfies $d_{\text {hor }}(x, z) \geq C$, then for any $\mathcal{T}$-geodesic $\omega$ with $|\omega|_{\mathcal{T}}=n t_{0}, n \geq 1$, and $\omega \cap \pi(s)=\{\pi(x)\}, d_{\text {hor }}(\omega x, \omega z) \geq \lambda^{n} d_{\text {hor }}(x, z)$.

Proof. If $|s|_{\text {vert }} \leq t_{0}$, the existence of the constants of quasi isometry, item (a) of Lemma 3.3 , and the definition of a $r$-vertical segment give an upper-bound for $d_{\text {hor }}(x, z)$. Let us thus assume $|s|_{\text {vert }}>t_{0}$. Choose $d$ such that $\lambda d-r^{\prime} \geq 2 r^{\prime}$, where $r^{\prime}$ is the above upperbound when $|s|_{\text {vert }}=t_{0}$. Then set $C=\max (d, M)$. Assume that $d_{\text {hor }}(x, z) \geq C$ and that $x$ and $z$ are exponentially separated in the direction given by $s$. If $[\pi(x), \pi(y)]=\omega_{0} \omega^{\prime}$ with $\left|\omega_{0}\right|_{\mathcal{T}}=t_{0}$, then $d_{\text {hor }}\left(\omega_{0} x, \omega_{0} z\right) \geq \lambda d_{\text {hor }}(x, z)$. Thanks to the inequality used to 
define $d$, one easily concludes that the horizontal distance between $s$ and the vertical tree through $y$ increases along $s$ when going from $x$ to $y$ which of course cannot happen. The conclusion follows from the exponential-separation property.

Proof of Proposition 4.7. We are given a $w$-corridor $\mathcal{C}, L$ the horizontal distance between two points $x$ and $y$ in $\mathcal{C}$, and $g$ a $(a, b)$-quasi geodesic in $\left(\mathcal{C}, d_{\text {tel }}^{v}\right)$ from a $v$-vertical tree through $x$ to a $v$-vertical tree through $y$ with $v \geq C_{4.4}(w)$. We assume that the $v$-vertical segments in $\mathcal{C}$ are exponentially separated. We consider the region $R$ with vertical width $C_{8.3}$ centered at the stratum $X_{\alpha}$ with $\alpha=\pi(x)$. We decompose $g$ in three subsets: the first one, denoted $g_{0}$, from the initial point of $g$ until the first point $z$ in $g \cap R$, the second one, denoted $g_{1}$, from $z$ to the last point $t$ in $g \cap R$, the third one, denoted $g_{2}$, from $t$ to the terminal point of $g$. Obviously $g_{1}$ can be approximated by the concatenation of two vertical segments with a horizontal geodesic in $X_{\alpha}$ (the approximation constant only depend on $L, a$ and $b$ ). We denote by $g_{1}^{\prime}$ the resulting set.

We now consider a maximal chain in $g_{0}$ which satisfies the following properties:

- its endpoints lie in a same stratum $X_{\beta}$,

- its image under $\pi$ does not intersect $[\alpha, \beta)$.

From Corollary 8.3, the endpoints of such a subchain are at horizontal distance smaller than $C_{8.3}$ one to each other. Thus, by substituting each such subchain by a horizontal geodesic connecting its endpoints, we construct a $C_{8.3}$-vertical segment $g_{0}^{\prime}$. We do the same thing for $g_{2}$, so obtaining a $C_{8.3}$-vertical segment $g_{2}^{\prime}$. ¿From Lemma 8.5, $g^{\prime}=$ $g_{0}^{\prime} \cup g_{1}^{\prime} \cup g_{2}^{\prime}$ lies in a bounded neighborhood of the $v$-vertical segments connecting its endpoints to $x_{1}$ and $x_{2}$. From the construction, $d_{t e l}^{H}\left(g, g^{\prime}\right) \leq a C_{8.3}+b+1$. The proposition follows.

\section{QUASICONVEXITY OF CORRIDORS}

In this section we prove Proposition 4.8, its adaptation to generalized corridors and Proposition 7.4.

9.1. Two basic lemmas. We need first a very general lemma about Gromov hyperbolic spaces.

Lemma 9.1. Let $(X, d)$ be a Gromov hyperbolic space. There exists $C \geq 0$ such that for any $r \geq C$ there is $D \geq 0$, increasing and affine in $r$, such that if $[x, y]$ is a diameter of a ball $\bar{B}_{x_{0}}(r)$, if $\omega$ is any chain in $X$ with $\omega \cap B_{x_{0}}(r)=\{x, y\}$, then $|\omega|_{d} \geq e^{D}$.

This lemma is a rewriting of Lemma 1.6 of [7].

Lemma 9.2. Let $\widetilde{X}$ be a tree of $\delta$-hyperbolic spaces which satisfies the exponentialseparation property. For any $v \geq C_{3.6}$, there exists $C \geq 0$ such that if $x, y, z, t$ are the vertices of a geodesic quadrilateral in some stratum $X_{\alpha}$, with $d_{\text {hor }}(x, z) \leq 2 \delta, d_{\text {hor }}(y, t) \leq 2 \delta$, and $d_{\text {hor }}(x, y) \geq C, d_{\text {hor }}(z, t) \geq C$, then for any $\mathcal{T}$-geodesic $\omega$ with $|\omega|_{\mathcal{T}} \geq C_{3.8}+n t_{0}$ and starting at $\pi(x)$, when considering the $v$-vertical segments over $\omega$ we have:

$$
d_{\text {hor }}(\omega x, \omega y) \geq \lambda^{n} d_{\text {hor }}(x, y) \Leftrightarrow d_{\text {hor }}(\omega z, \omega t) \geq \lambda^{n} d_{\text {hor }}(z, t)
$$

Proof. If $A, B$ are two subsets of a metric space $(X, d)$, we set $d^{s}(A, B)=\sup _{x \in A, y \in B} d(x, y)$. Let us consider any $\mathcal{T}$-geodesic $\omega$ with $|\omega|_{\mathcal{T}}=t_{0}$ starting at $\alpha$. From Lemma 3.3,

$$
d_{h o r}^{s}(\omega x, \omega z) \leq \lambda_{+}^{t_{0}}(2 \delta+\mu)
$$


and

$$
d_{\text {hor }}^{s}(\omega y, \omega t) \leq \lambda_{+}^{t_{0}}(2 \delta+\mu) .
$$

Assume $d_{\text {hor }}(\omega x, \omega y) \geq \lambda d_{\text {hor }}(x, y)$ but $d_{\text {hor }}(\omega z, \omega t)<\lambda d_{\text {hor }}(z, t)$.

We take $d_{\text {hor }}(x, y) \geq M$ and $d_{\text {hor }}(z, t) \geq M$. Assume $d_{\text {hor }}^{s}(\omega z, \omega t) \leq \frac{1}{\lambda} d_{\text {hor }}(z, t)$. But $d_{\text {hor }}(z, t) \leq 4 \delta+d_{\text {hor }}(x, y)$. Putting together these inequalities we get

$$
\lambda d_{\text {hor }}(x, y) \leq 2 \lambda_{+}^{t_{0}}(2 \delta+\mu)+\frac{1}{\lambda}\left(4 \delta+d_{\text {hor }}(x, y)\right) .
$$

Whence an upper bound for $d_{h o r}(x, y)$ and thus for $d_{h o r}(z, t)$. If $d_{h o r}^{s}(\omega z, \omega t)>\frac{1}{\lambda} d_{h o r}(z, t)$ then the lemma follows from the definition of the constant $C_{3.8}$, see the corresponding lemma.

The above two lemmas are not needed if one only considers trees of 0-hyperbolic spaces, the proof in this last case being much simpler.

\subsection{Approximation of quasi geodesics with bounded vertical deviation.}

Lemma 9.3 below states that in a tree of hyperbolic spaces $(\widetilde{X}, \mathcal{T})$ a quasi geodesic with bounded image in $\mathcal{T}$ lies close to a corridor between its endpoints. This is intuitively obvious and nothing is new neither surprising in the arguments of the proof: they heavily rely upon the $\delta$-hyperbolicity of the strata and the fact that strata are quasi isometrically embedded into each other. For the sake of brevity, we do not develop them here.

Lemma 9.3. Let $(\widetilde{X}, \mathcal{T}, \pi)$ be a tree of hyperbolic spaces. For any $\kappa, b \geq 0, a \geq 1$ and $v \geq C_{3.6}$ there exists $C \geq 0$ such that if $g$ is any $(a, b)$-quasi geodesic of $\widetilde{X}$ with $\operatorname{diam}_{\mathcal{T}}(\pi(g)) \leq \kappa$, if $\mathcal{C}$ is a generalized $v$-corridor whose vertical boundaries pass through the endpoints of $g$ then $g \subset \mathcal{N}_{\widetilde{X}}^{C}(\mathcal{C})$.

9.3. Stairs. Notations: The sign $\simeq_{1}$ stands for an equality up to $\pm 1,(\widetilde{X}, \mathcal{T}, \pi)$ a tree of hyperbolic spaces which satisfies the exponential-separation property, $v \geq C_{3.6}$ a constant.

Definition 9.4. Let $r \geq M$. A $r$-stair relative to a generalized $v$-corridor $\mathcal{C}$ is a $v$ telescopic chain $\mathcal{S}$ the vertical segments of which have vertical length greater than $C_{3.8}$ and such that, for any horizontal geodesic $\left[a_{i}, b_{i}\right]$ in $\mathcal{S}$ :

(a) $d_{\text {hor }}\left(a_{i}, b_{i}\right) \geq r$ and $d_{\text {hor }}\left(\left[a_{i}, b_{i}\right], \mathcal{C}\right) \simeq_{1} d_{\text {hor }}\left(a_{i}, P_{\mathcal{C}}^{\text {hor }}\left(a_{i}\right)\right)$,

(b) any two points $a, b \in\left[a_{i}, b_{i}\right]$ with $d_{\text {hor }}(a, b) \geq r$ are exponentially separated in the direction of the $\mathcal{T}$-geodesic $\left[\pi\left(a_{i}\right), \pi\left(a_{i+1}\right)\right]$.

See Figure 4.

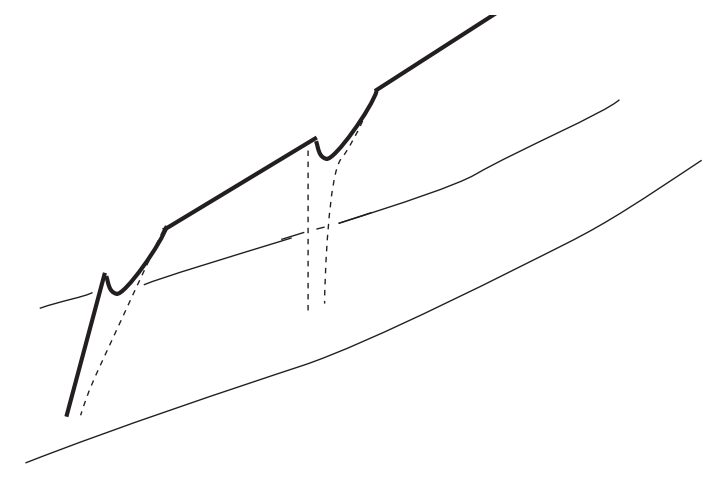

Figure 4. A stair 
Lemma 9.5. With the notations of Definition 9.4: there exist $C \geq C_{9.2}$ such that for any $r \geq C$, if $\mathcal{C}$ is a generalized $v$-corridor, if $\mathcal{S}$ is a $r$-stair relative to $\mathcal{C}$, if $\mathcal{U}$ is a generalized $v$-corridor between a vertical tree through the terminal point of $\mathcal{S}$ and a vertical boundary of $\mathcal{C}$, then

$$
\mathcal{S} \subset \mathcal{N}_{h o r}^{r+2 \delta}(\mathcal{U})
$$

Proof. Let $a_{i}, b_{i} \in \mathcal{S}$ as given in Definition 9.4 and let $z$ be a point at the intersection of the stratum $X_{\pi\left(a_{i}\right)}$ with a vertical tree through some point farther in the stair. Then:

Claim 1: There exists $K>0$ not depending on $a_{i}$ nor $z$ such that, if $r$ is sufficiently large then $d_{\text {hor }}\left(\left[a_{i}, z\right], \mathcal{C}\right) \geq d_{\text {hor }}\left(a_{i}, P_{\mathcal{C}}^{\text {hor }}\left(a_{i}\right)\right)-K$.

Proof of Claim 1: Choose $K$ such that $e^{D_{9.1}(K)}>4 \delta+1$ and assume $d_{\text {hor }}\left(\left[a_{i}, z\right], \mathcal{C}\right)<$ $d_{\text {hor }}\left(a_{i}, P_{\mathcal{C}}^{\text {hor }}\left(a_{i}\right)\right)-K$. Then Lemma 9.1 implies that $\left[b_{i}, z\right]$ descends at least until a $2 \delta$-neighborhood of $a_{i}$. Assume $r \geq C_{9.2}+2 \delta$. Then Lemma 9.2 gives an initial segment of $\left[b_{i}, z\right]$ of horizontal length greater than $r-2 \delta$ which is dilated in the direction of $\left[\pi\left(a_{i}\right), \pi\left(a_{i+1}\right)\right]$. If $r$ is chosen sufficiently large with respect to the constants of hyperbolicity for a corridor (see Lemma 3.7), we get $z^{\prime}$ at the intersection of the considered vertical tree through $z$ with the stratum $X_{\pi\left(a_{i+1}\right)}$ such that $d_{h o r}\left(\left[a_{i+1}, z^{\prime}\right], \mathcal{C}\right)<$ $d_{\text {hor }}\left(a_{i+1}, P_{\mathcal{C}}^{\text {hor }}\left(a_{i+1}\right)\right)-K$. The repetition of these arguments show that the horizontal distance between $\mathcal{S}$ and the vertical tree through $z$ does not decrease along $\mathcal{S}$. This is an absurdity since $z$ was chosen in a vertical tree through a point farther in $\mathcal{S}$. The proof of Claim 1 is complete.

Claim 2: There exists $K(r)$ not depending on $b_{i}$ nor $z$ such that, if $r$ is sufficiently large then $d_{\text {hor }}\left(\left[b_{i}, z\right], \mathcal{C}\right) \geq d_{\text {hor }}\left(b_{i}, P_{\mathcal{C}}^{\text {hor }}\left(b_{i}\right)\right)-K(r)$.

Proof of Claim 2: Let $z_{\star} \in\left[b_{i}, z\right]$ with $d_{\text {hor }}\left(z_{\star}, P_{\mathcal{C}}^{\text {hor }}\left(z_{\star}\right)\right) \simeq_{1} \max \left(d_{\text {hor }}\left(\left[b_{i}, z\right], \mathcal{C}\right), d_{\text {hor }}\left(a_{i}\right.\right.$, $\left.\left.P_{\mathcal{C}}^{\text {hor }}\left(a_{i}\right)\right)\right)$. From the $\delta$-hyperbolicity of the strata, $\left[b_{i}, z_{\star}\right]$ lies in the horizontal $2 \delta$ neighborhood of $\left[a_{i}, b_{i}\right]$. Assume $d_{h o r}\left(b_{i}, z_{\star}\right) \geq r$ and is sufficiently large to apply Lemma 9.2. Then there is $K(r)$ such that, if $z_{\star}$ satisfies $d_{\text {hor }}\left(z_{\star}, P_{\mathcal{C}}^{\text {hor }}\left(z_{\star}\right)\right)<d_{\text {hor }}\left(b_{i}, P_{\mathcal{C}}^{\text {hor }}\left(b_{i}\right)\right)-$ $K(r)$, the points $b_{i}$ and $z_{\star}$ are exponentially separated in the direction of $\left[\pi\left(a_{i}\right), \pi\left(a_{i+1}\right)\right]$. We thus obtain at $a_{i+1}$ a situation similar to that of Claim 1. The proof of Claim 2 follows.

Lemma 9.5 is easily deduced from the above two claims, we leave the reader work out the easy details.

Lemma 9.6. For any $r \geq C_{9.5}$ there exists $C>0$ such that, if $\mathcal{C}$ is a generalized $v$-corridor, if $\mathcal{S}$ is a r-stair relative to $\mathcal{C}$ which is not contained in the vertical $C$ neighborhood of the stratum containing its initial point, then the terminal point of $\mathcal{S}$ does not belong to the r-neighborhood of $\mathcal{C}$ in $\widetilde{X}$.

Proof. Decompose $\mathcal{S}$ in maximal substairs $\mathcal{S}_{0} \cdots \mathcal{S}_{k}$ such that $\pi\left(\mathcal{S}_{j}\right)$ is a geodesic of $\mathcal{T}$. Let $\left[a_{i}, b_{i}\right]$ be the first horizontal geodesic in $\mathcal{S}_{j}$, let $x$ be the initial point of $\mathcal{S}_{j}$ and let $z$ be any point in $\mathcal{S}_{j}$ with $n t_{0} \leq d_{\mathcal{T}}(\pi(z), \pi(x)) \leq(n+1) t_{0}$.

The inequality

$$
d_{\text {hor }}\left(z, P_{\mathcal{C}}^{\text {hor }}(z)\right) \geq C t e \lambda^{n} d_{\text {hor }}\left(a_{i}, b_{i}\right)
$$

is an easy consequence of the definition of a stair and of Lemma 9.2 as soon as $r \geq C_{9.2}$. Indeed, the initial segment of horizontal length $r$ in $\left[b_{i}, P_{\mathcal{C}}^{h o r}\left(b_{i}\right)\right]$ lies in the horizontal $2 \delta$-neighborhood of $\left[b_{i}, a_{i}\right]$. The assertion then follows from item (b) of Definition 9.4 and Lemma 9.2.

The inequality (2) readily gives the announced result. 


\subsection{Approximation of a quasi geodesic by a stair.}

Notations: $(\widetilde{X}, \mathcal{T})$ a tree of $\delta$-hyperbolic spaces which satisfies the exponential-separation property, $v \geq C_{3.6}$.

Lemma 9.7. For any $a \geq 1, b \geq 0$ there exists $D \geq 0$ such that for any $r \geq D$ there are $C, E \geq 0$, where $E$ is affine in $r$, such that if $\mathcal{C}$ is a generalized $v$-corridor, if the endpoints of a $v$-telescopic $(a, b)$-quasi geodesic $g$ are in a horizontal $r$-neighborhood of $\mathcal{C}$, if $g$ lies in the closed complement of this horizontal neighborhood and if the vertical segments in $g$ have vertical length greater than $3\left(C_{3.8}+D_{8.1}\right)$ then either $g$ lies in the $C$-neighborhood of a E-stair relative to $\mathcal{C}$ or $g$ is contained in the $C$-neighborhood of $\mathcal{C}$.

Proof. We decompose the proof in two steps. The first one is only a warm-up, to present the ideas in a particular, but important, case. The general case, detailed in the second step, is technically more involved but no new phenomenon appears.

Step 1: Proof of Lemma 9.7 when the horizontal length of any horizontal path in $g$ is greater than some constant (depending on a et $b$ ). The endpoints of any horizontal path $h$ in $g$ with horizontal length greater than $C_{8.1}$ are exponentially separated under every geodesic $\omega$ of $\mathcal{T}$ with length $D_{8.1}$. If $|h|_{\text {hor }} \geq C_{9.2}$, this is also true for any horizontal geodesic $h^{\prime}$ in the $2 \delta$-neighborhood of $h$. Finally, if $|h|_{h o r}$ is sufficiently large, by Lemma 3.7 the endpoints of $h$ are also exponentially separated in any $v$-corridor containing $h$. If $e(a, b)$ (we do not indicate the dependance on $v$ ) is the maximum of the above constants, we now assume $|h|_{\text {hor }} \geq 3 e(a, b)$.

Let us consider two consecutive horizontal geodesics $h_{1}, h_{2}$ in $g$, separated by a vertical segment $s$. Let $\mathcal{D}$ be a corridor containing $h_{1}$ and $s$. Then:

$$
\left|h_{2} \cap \mathcal{N}_{\text {hor }}^{2 \delta}(\mathcal{D})\right|_{\text {hor }} \leq e(a, b) .
$$

Otherwise we have a contradiction with the fact that the endpoints of any subgeodesic of $h_{2}$ whose length is greater than $C_{8.1}$ are exponentially separated in the direction of $h_{1}$.

From the inequality (3), the concatenation of $h_{1}, s$ and $h_{2}$ is $e(a, b)$-close, with respect to the horizontal distance, of a $2 e(a, b)$-stair relative to $\mathcal{C}$ if $d_{\text {hor }}\left(h_{1}, \mathcal{C}\right) \simeq_{1} d_{\text {hor }}\left(a_{1}, P_{\mathcal{C}}^{\text {hor }}\left(a_{1}\right)\right)$ where $a_{1}$ is the initial point of $h_{1}$.

Let us now set $r \geq 3 e(a, b)$ and assume that the horizontal geodesics in $g$ have horizontal length greater than $r$. Let $x$ be the initial point of $g$ (in particular $\left.d_{h o r}\left(x, P_{\mathcal{C}}^{h o r}(x)\right) \simeq_{1} r\right)$. Let $s$ be the vertical segment starting at $x$ and ending at $y$ in $g$. Let $h$ be the horizontal geodesic following $s$ along $g$. Let $n \geq 1$ be the greatest integer with $n\left(C_{3.8}+D_{8.1}\right) \leq|s|_{\text {vert }}$.

By assumption $x$ and $P_{\mathcal{C}}^{h o r}(x)$ are exponentially separated in the direction of $s$. Since the strata are quasi isometrically embedded one into each other, this gives $\kappa>1$ such that, any two points $p, q \in\left[x, P_{\mathcal{C}}^{\text {hor }}(x)\right]$ with $d_{\text {hor }}(p, q) \geq \max \left(\frac{1}{\kappa} r, M\right)$ satisfy $d_{\text {hor }}(\pi(s) p, \pi(s) q) \geq$ $\lambda^{n} d_{h o r}(p, q)$. Thus the same arguments as those exposed above when working with $h_{1}, h_{2}$ show that $\left|h \cap \mathcal{N}_{\text {hor }}^{2 \delta}\left(\left[y, P_{\mathcal{C}}^{h o r}(y)\right]\right)\right|_{\text {hor }} \leq \max \left(e(a, b), \frac{1}{\lambda^{n} \kappa} r, M\right)$. If $n$ is greater than some critical constant $n_{*}$, this last maximum is equal to $e(a, b)$. Thus, in this case we take $h_{1}=\left[x, P_{\mathcal{C}}^{h o r}(x)\right]$ and $h_{2}=h$ : the above arguments prove that the concatenation of $h_{1}, s$ and $h_{2}$ is $e(v, a, b)$-close to a $e(a, b)$-stair. If $n$ is smaller than $n_{*}$, then we substitute $r$ by $\lambda_{+}^{n_{*}\left(C_{3.8}+D_{8.1}\right)} r$, modify $g$ by taking the starting point at the endpoint $y$ of $s$ and take $h_{1}$ as the first horizontal geodesic.

In both cases, by repeating the arguments above at any two consecutive horizontal geodesic following the first two ones along $g$, we show that $g$ is $e(a, b)$-close, with respect 
to the horizontal distance, of a $e(a, b)$-stair relative to $\mathcal{C}$.

Step 2: Adaptation of the argument to the general case: The boundary trees of $\mathcal{C}$ are denoted by $L_{1}$ and $L_{2}$, and $g$ goes from $L_{1}$ to $L_{2}$. We choose a positive constant $r$, which when necessary will be set sufficiently large with respect to the constants $C_{9.5}$, $M, \delta$ and $C_{9.2}$. Let $x_{0}$ be the initial point of $g$. It lies in the boundary of the horizontal $r$-neighborhood of $\mathcal{C}$. We denote by $\mathcal{C}_{i}$ and $x_{i}, i=1, \cdots$, a sequence of corridors and points of $g$ defined inductively as follows:

(a) $\mathcal{C}_{i}$ is a corridor with boundary trees a $v$-vertical tree through $x_{i-1}$ and the $v$-vertical boundary $L_{2}$ of $\mathcal{C}$

(b) $x_{i}$ is the first point following $x_{i-1}$ along $g$ such that $d_{h o r}\left(x_{i}, P_{\mathcal{C}_{i}}^{\text {hor }}\left(x_{i}\right)\right) \geq r$.

The chain in $g$ between $x_{i-1}$ and $x_{i}$ is denoted by $g_{i-1, i}$. Obviously $g_{i-1, i}$ is contained in the horizontal $r$-neighborhood of $\mathcal{C}_{i}$. We project it to $\mathcal{C}_{i}$. From Lemma 4.10, we get a $D_{4.10}$-telescopic $\left(C_{4.10}, C_{4.10}\right)$-quasi geodesic of $\left(\mathcal{C}_{i}, d_{\text {tel }}^{D_{4.10}}\right)$. We set $X(a, b, r)=$ $C_{4.7}\left(r, C_{4.10}, C_{4.10}\right)$. From Proposition 4.7, $P_{\mathcal{C}_{i}}^{\text {hor }}\left(g_{i-1, i}\right)$ is contained in the $X(a, b, r)$ neighborhood of the concatenation of a subpath of $\left[x_{i-1}, P_{\mathcal{C}_{i-1}}^{\text {hor }}\left(x_{i-1}\right)\right]$ with a vertical segment in $\mathcal{C}_{i}$ (and is followed by $\left[P_{\mathcal{C}_{i}}^{\text {hor }}\left(x_{i}\right), x_{i}\right]$ ). Consider in this approximation of (a subchain of) $g$ a maximal collection of points $y_{i}$ which defines a $r$-stair relative to $\mathcal{C}$. The points $y_{i}$ do not necessarily agree with the $x_{i}$ 's, because it might happen that, after $x_{i-1}$ for instance, the approximation constructed above reenters in the $r$-neighborhood of $\mathcal{C}_{i-1}$ before leaving the $r$-neighborhood of $\mathcal{C}_{i}$. We proceed as in Step 1 and choose the $y_{i}$ 's so that:

(a) either $y_{i}$ is contained in a horizontal geodesic of the chain, and from the observations in Step 1, this horizontal geodesic may be included in a stair,

(b) or the vertical distance from $y_{i}$ to the next horizontal geodesic is at least $C_{3.8}+D_{8.1}$.

Either we obtain a non-trivial $r$-stair relative to $\mathcal{C}$ which approximates a subchain $g_{0}^{\prime}$ of $g$ or the approximation we constructed above exhausts $g$ and is contained in some telescopic neighborhood of $\mathcal{C}$ the size of which is obtained from the previously exhibited constants. In this last case, the same assertion holds for the whole $g$. This is one of the announced alternatives.

We can thus assume that we got $y_{0}, \cdots, y_{k}$ forming a $r$-stair relative to $\mathcal{C}$. It is denoted by $S$. Since the strata are quasi isometrically embedded one into each other, there is $\kappa>1$, only depending on the constants of quasi isometry, such that $S$ is in fact a $\max \left(\frac{1}{\kappa} r, M, e(a, b)\right)$-stair relative to $\mathcal{C}$. As soon as $r>\kappa(M+e(a, b))$, which we suppose from now, this maximum is just $\frac{1}{\kappa} r$. Thus $S$ is a $\frac{r}{\kappa}$-stair whose horizontal geodesics have horizontal length at least $r$.

By construction $S$ approximates $g_{0}^{\prime} \subset g$. We now consider the maximal subchain $g_{1}^{\prime}$ of $g$ starting at (or near - recall that we constructed an approximation of a subchain of $g$ ) $y_{k}$ which lies in the $r$-neighborhood of $\mathcal{C}_{k}$. This last corridor plays the rôle of the corridor $\mathcal{U}$ of Lemma 9.5. We project the subchain $g_{1}^{\prime}$ to $\mathcal{C}_{k}$, so getting a $\left(C_{4.10}, C_{4.10}\right)$-quasi geodesic of this corridor. From Lemma 9.5, and because of the hyperbolicity of the strata, each horizontal geodesic of the $\frac{r}{\kappa}$-stair $S$ admits a subgeodesic with horizontal length greater than $\frac{\kappa-1}{\kappa} r$ in the horizontal $2 \delta$-neighborhood of $\mathcal{C}_{k}$. If $r$ is chosen sufficiently large, Lemma 9.2 gives horizontal geodesics in $\mathcal{C}_{k}$ with horizontal length greater than $M$ which are dilated in the same directions than the horizontal geodesics of $S$. Now Proposition 4.7 applies and allows us to approximate the projection of $g_{1}^{\prime}$ on $\mathcal{C}_{k}$ by a sequence of these horizontal geodesics. But each one of these horizontal geodesics is close to a point in $g_{0}^{\prime} \subset g$. Thus, since $g$ is a $(a, b)$-quasi geodesic, the vertical length of $g_{1}^{\prime}$, and so its 
telescopic length, is bounded above by a constant depending on $a$ and $b$. So we can forget $g_{1}^{\prime}$ and continue the construction of our $\frac{r}{\kappa}$-stair relative to $\mathcal{C}$ at the point where the approximation of $g_{1}^{\prime}$ leaves the $r$-neighborhood of $\mathcal{C}_{k}$. We eventually exhaust $g$ and obtain a $\frac{r}{\kappa}$-stair relative to $\mathcal{C}$.

9.5. Proof of Proposition 4.8. Let $g$ and $\mathcal{C}$ be as given by this proposition. Assume that some subchain $g^{\prime}$ of $g$ leaves and then reenters the horizontal $D_{9.7}$-neighborhood of $\mathcal{C}$. Assume that $g^{\prime}$ is not contained in the telescopic $C_{9.7}\left(D_{9.7}, a, b\right)$-neighborhood of $\mathcal{C}$. We set $C_{9.7} \equiv C_{9.7}\left(D_{9.7}, a, b\right)$ and $E_{9.7} \equiv E_{9.7}\left(D_{9.7}, a, b\right)$.

Suppose for the moment that the vertical segments in $g^{\prime}$ have vertical length greater than $3\left(C_{3.8}+D_{8.1}\right)$. Then Lemma 9.7 gives $G$, a $E_{9.7}$-stair relative to $\mathcal{C}$ with $d_{\text {tel }}^{H}\left(g^{\prime}, G\right) \leq$ $C_{9.7}$. From Lemma 9.6, $G$ does not leave the vertical $C_{9.6}\left(E_{9.7}\right)$-neighborhood of the stratum containing the initial point of $G$. Therefore, by setting $V(a, b)=C_{9.6}\left(E_{9.7}\right)+C_{9.7}$, $g^{\prime}$ does not leave the vertical $V(a, b)$-neighborhood of this stratum. From Lemma 9.3, $g^{\prime}$ lies in the telescopic $C_{9.3}(V(a, b), a, b)$-neighborhood of $\mathcal{C}$.

It remains to consider the case where the vertical segments in $g^{\prime}$ are not sufficiently large. Let $s$ be a vertical segment in $g$ with $|s|_{\text {vert }}<X \equiv 3\left(C_{3.8}+D_{8.1}\right)$.

$(\dagger)$ Thanks to the assumption that all the attaching-maps of the tree of hyperbolic spaces are quasi isometries, $s$ is contained in a vertical segment $s^{\prime}$ of vertical length greater than $X$. We modify $g^{\prime}$ by sliding, along $s^{\prime}$, a horizontal geodesic in $g^{\prime}$ incident to $s$ until getting a vertical segment with vertical length $X$. This yields a new telescopic $\left(a^{\prime}, b^{\prime}\right)$-quasi geodesic in a bounded neighborhood of $g$, where the constants $a^{\prime}, b^{\prime}$ only depend on $a, b$ and on the constants of quasi isometry. After finitely many such moves, we obtain a quasi geodesic as desired, and we are done. Since the vertical distance between two strata is uniformly bounded away from zero, after finitely many such substitutions, we eventually get a quasi geodesic, in a bounded neighborhood of $g$, which satisfies the assumptions required by Lemma 9.7. This completes the proof of Proposition 4.8.

9.6. Adaptation to generalized corridors. The only problem is to get a telescopic chain with vertical segments sufficiently large. We start from the sentence marked by a $(\dagger)$ in the preceding subsection. If $s$ is not contained in a vertical segment $s^{\prime}$ of vertical length greater than $X$, we obtain a vertical segment $\mathbf{s}$ from $b_{i}$ to $a_{i+1}$ satisfying the following properties (we still denote by $g^{\prime}$ the $\left(a^{\prime}, b^{\prime}\right)$-quasi geodesic eventually obtained, we denote by $\mathbf{s}_{0}$ the vertical segment of $g^{\prime}$ ending at $a_{i}$ and by $\mathbf{s}_{1}$ the one starting at $\left.b_{i+1}\right)$ :

(a) there is no vertical segment starting at $a_{i}$ (resp. at $a_{i+1}$ ) over the edge $\pi(\mathbf{s})$ (resp. over $\left.\pi\left(\mathbf{s}_{1}\right)\right)$

(b) there is no vertical segment ending at $b_{i}$ over $\pi\left(\mathbf{s}_{0}\right)$.

Consider horizontal geodesics $\alpha_{i}=\left[a_{i}, P_{\mathcal{C}}^{h o r}\left(a_{i}\right)\right], \beta_{i}=\left[b_{i}, P_{\mathcal{C}}^{\text {hor }}\left(b_{i}\right)\right], \alpha_{i+1}=\left[a_{i+1}\right.$, $\left.P_{\mathcal{C}}^{\text {hor }}\left(a_{i+1}\right)\right]$ and $\beta_{i+1}=\left[b_{i+1}, P_{\mathcal{C}}^{\text {hor }}\left(b_{i+1}\right)\right]$. By the $\delta$-hyperbolicity of the strata, there is $a_{i}^{\prime} \in\left[a_{i}, b_{i}\right] \cap \mathcal{N}_{\text {hor }}^{2 \delta}\left(\alpha_{i} \cup \beta_{i}\right)$ and $b_{i}^{\prime} \in\left[a_{i+1}, b_{i+1}\right] \cap \mathcal{N}_{\text {hor }}^{2 \delta}\left(\alpha_{i+1} \cup \beta_{i+1}\right)$. Because the strata are quasi isometrically embedded one into each other, we get two points $a_{i}^{\prime \prime}, b_{i}^{\prime \prime}$ which satisfy:

(A) they are $Y$-close (with respect to the horizontal distance) respectively to $a_{i}^{\prime}$ and $b_{i}^{\prime}$, where the constant $Y$ only depends on $\delta$ and on the constants of quasi isometry;

(B) there is a $v$-vertical segment from $a_{i}^{\prime \prime}$ to $b_{i}^{\prime \prime}$ which is contained in a larger $v$-vertical segment going over $\pi\left(\mathbf{s}_{0}\right)$ and $\pi\left(\mathbf{s}_{1}\right)$.

We modify $g^{\prime}$ by going from $a_{i}$ to $a_{i}^{\prime \prime}$ then to $b_{i}^{\prime \prime}$ and eventually end at $b_{i+1}$. The resulting chain is a $\left(a^{\prime \prime}, b^{\prime \prime}\right)$-quasi geodesic, where the constants $a^{\prime \prime}, b^{\prime \prime}$ only depends on $\delta$ and on the constants of quasi isometry. Moreover this new chain is in a bounded neighborhood 
of $g^{\prime}$. Thanks to item (B), we can modify it by enlarging the vertical segment from $a_{i}^{\prime \prime}$ to $b_{i}^{\prime \prime}$. The conclusion in then the same as in the preceding subsection.

9.7. Proof of Proposition 7.4. The arguments are similar to those exposed for proving the quasi convexity of the corridors. We give here only a sketch of the proof. Because a tree of cone-vertices is a vertical tree, the horizontal deviation of a tree of cone-vertices with respect to $\mathcal{C}$ depends linearly on the vertical variation of the orbit. Thus, if a sufficiently large segment of the orbit remains outside a sufficiently large horizontal neighborhood of $\mathcal{C}$, the exponential separation implies that the horizontal distance between the orbit and $\mathcal{C}$ exponentially increases with the vertical length of the orbit. Assume now that the exceptional orbit considered is followed by another one. The strong exponential separation gives the same consequence: this second exceptional orbit does not go back to $\mathcal{C}$ and the horizontal distance with respect to $\mathcal{C}$ exponentially increases with its vertical length, as soon as this length is sufficiently large. Here the arguments are similar to those used for proving Lemmas 9.5 and 9.6. Finally, if the exceptional orbit is followed by a quasi geodesic in $\widehat{X}$, then the approximation by a stair as was done before, yields the same conclusion.

\section{REFERENCES}

[1] E. ALIBEGOVIC 'A combination theorem for relatively hyperbolic groups', Bulletin of the London Mathematical Society (3) 37 (2005) 459-466.

[2] M. BESTVINA and M. FEIGHN 'A combination theorem for negatively curved group' Journal of Differential Geometry (1) 35 (1992) 85-101. With an addendum and correction Journal of Differential Geometry (4) 43 (1996) 783-788.

[3] B.H. BOWDITCH 'Relatively hyperbolic groups', preprint, University of Southampton 1999 (http://www.warwick.ac.uk/ masgak/preprints.html).

[4] B.H. BOWDITCH 'Stacks of hyperbolic spaces and ends of 3-manifolds', preprint, University of Southampton 2002 (http://www.warwick.ac.uk/ masgak/preprints.html).

[5] M. BRIDSON and A. HAEFLIGER Metric spaces of non-positive curvature Grundlehren der Mathematischen Wissenschaften 319, Springer-Verlag (1999).

[6] I. BUMAGIN 'On definitions of relatively hyperbolic groups', Geometric methods in group theory Contemporary Mathematics series 372 (2005).

[7] M. COORNAERT, T. DELZANT and A. PAPADOPOUlOS Géométrie et théorie des groupes Lecture Notes in Math. 1441, Springer Verlag (1990).

[8] F. DAHMANI 'Combination of convergence groups' Geometry and Topology 7 (2003) 933-963.

[9] B. FARB 'Relatively hyperbolic groups' Geom. Funct. Anal. (GAFA) 8 (1998) 1-31.

[10] F. GAUTERO 'Hyperbolicity of mapping-torus groups and spaces' L'Enseignement mathématique, 49 (2003) 263-305.

[11] . F. GAUTERO Quatre problèmes dynamiques, géométriques ou algébriques autour de la suspension. Habilitation thesis, Université Blaise Pascal, Clermont-Ferrand (2006).

[12] F. GAUTERO and M. LUSTIG 'Relative hyperbolization of (one-ended hyperbolic)-by-cyclic groups' Math. Proc. Camb. Phil. Soc. 137 (2004) 595-611.

[13] R. GITIK 'On the combination theorem for negatively curved groups' International Journal of Algebra Comput. (6) 6 (1996) 751-760.

[14] M. GROMOV 'Hyperbolic groups', Essays in Group Theory Math. Sci. Res. Inst. Publ. 8, Springer 1987, 75-263.

[15] I. KAPOVICH 'A non-quasiconvexity embedding theorem for hyperbolic groups' Mathematical Proceedings of the Philosophical Cambridge Society 127 (1999) 461-486.

[16] I. KAPOVICH 'Mapping tori of endomorphisms of free groups' Communications in Algebra (6) 28 (2000) 2895-2917.

[17] O. KHARLAMPOVICH and A. MYASNIKOV 'Hyperbolic groups and free constructions' Transactions of the American Mathematical Society (2) 350 (1998) 571-613. 
[18] M. MJ and L. REEVES 'A combination theorem for strong relative hyperbolicity', Geometry and Topology 12 (3) (2008) 1777-1798.

[19] D. OSIN 'Weak hyperbolicity and free constructions' Contemporary Mathematics 360 (2004) 103111.

[20] D. OSIN 'Relatively hyperbolic groups: Intrinsic geometry, algebraic properties and algorithmic problems' Memoirs of the American Mathematical Society (843) 179 (2006).

[21] D. OSIN 'Relative Dehn functions of amalgated products and HNN extensions' Contemporary Mathematics 394 (2006) 209-220.

[22] A. SZCZEPAŃSKI 'Relatively hyperbolic groups' Michigan Math. Journal 45 (1998) 611-618.

Université de Nice Sophia Antipolis, Parc Valrose, Laboratoire de Mathématiques J.A. Dieudonné, UMR CNRS 7351, 06108 Nice Cedex 02, France

E-mail address: Francois.Gautero@unice.fr 